\title{
Probabilistic Finite Element Modeling of Waste Rollover
}

M. A. Khaleel

W. F. Cofer ${ }^{(a)}$

A. A. Al-fouqaha ${ }^{(a)}$

September 1995

Prepared for

the U.S. Department of Energy

under Contract DE-AC06-76RLO 1830

Pacific Northwest Laboratory

Richland, Washington 99352

(a) Department of Civil and Environmental Engineering Washington State University

Pullman, Washington 99164-2910 


\section{DISCLAIMER}

This report was prepared as an account of work sponsored by an agency of the United States Government. Neither the United States Government nor any agency thereof, nor any of their employees, make any warranty, express or implied, or assumes any legal liability or responsibility for the accuracy, completeness, or usefulness of any information, apparatus, product, or process disclosed, or represents that its use would not infringe privately owned rights. Reference herein to any specific commercial product, process, or service by trade name, trademark, manufacturer, or otherwise does not necessarily constitute or imply its endorsement, recommendation, or favoring by the United States Government or any agency thereof. The views and opinions of authors expressed herein do not necessarily state or reflect those of the United States Government or any agency thereof. 


\section{DISCLAIMER}

Portions of this document may be illegible in electronic image products. Images are produced from the best available original document. 


\section{Executive Summary}

Stratification of the wastes in many Hanford storage tanks has resulted in sludge layers which are capable of retaining gases formed by chemical and/or radiolytic reactions. As the gas is produced, the mechanisms of gas storage evolve until the resulting buoyancy in the sludge leads to instability, at which point the sludge "rolls over" and a significant volume of gas is suddenly released. Because the releases may contain flammable gases, these episodes of release are potentially hazardous. Mitigation techniques are desirable for more controlled releases at more frequent intervals.

To aid the mitigation efforts, a methodology for predicting the probability of sludge rollover at specific times is desired. This methodology would then provide a rational basis for the development of a schedule for the mitigation procedures. In addition, a knowledge of the sensitivity of the sludge rollovers to various physical and chemical properties within the tanks would provide direction for efforts to reduce the frequency and severity of these events. In this report, the use of probabilistic finite element analyses for computing the probability of rollover and the sensitivity of rollover probability to various parameters is described.

The structural reliability program, CALREL, was applied to the analysis of the sludge of a typical tank. Four significant parameters (supernatant density, reference pressure, initial void fraction, and supernatant depth) were chosen to be random, with assumed statistical distributions. The history of gas generation and the resulting net density distribution were defined using elements of the BOND6 computer program. From the resulting net densities, the stress within the sludge was obtained via an axisymmetric finite element analysis. The initiation of sludge rollover was designated as the limit state, related to the assumed strength of the material. Using the First Order Reliability Method (FORM), a design point was obtained for the analyses corresponding to discrete gas generation steps. The probability of failure, the reliability index, and the sensitivity with respect to each random variable were then computed.

Based on the above model conditions, the results showed that the mechanism of gas release changes with time from a small release at the top of the sludge to a rollover from gas trapped below. Also, as expected, the probability of failure increases and the reliability diminishes over time. The addition of sludge strength was shown to provide a significant increase in the reliability of the system at all steps. Finally, the reliability of the system was shown to be most sensitive to the supernatant density and, to a lesser degree, to the reference pressure within the tank. The reliability was relatively insensitive to the initial void fraction at the top of the sludge layer and to the depth of the supernatant. 



\section{Contents}

Executive Summary $\ldots \ldots \ldots \ldots \ldots \ldots \ldots \ldots \ldots \ldots \ldots \ldots \ldots \ldots$ ii

1.0 Introduction $\ldots \ldots \ldots \ldots \ldots \ldots \ldots \ldots \ldots \ldots \ldots \ldots \ldots \ldots \ldots \ldots \ldots \ldots$

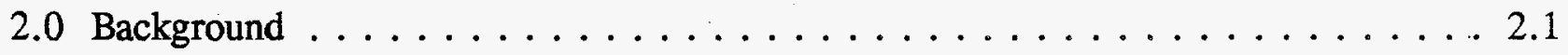

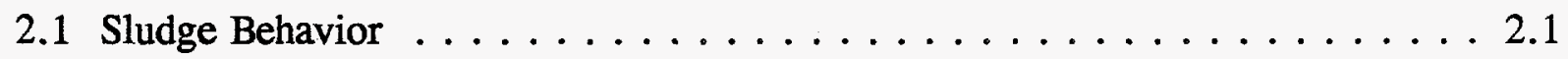

2.2 Structural Reliability Analysis Program $\ldots \ldots \ldots \ldots \ldots . \ldots \ldots$

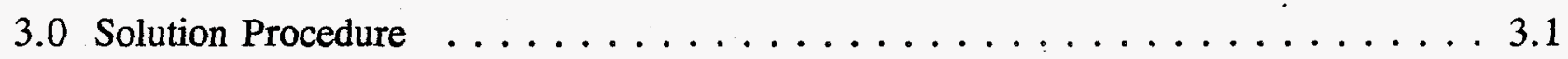

3.1 Finite Element Software $\ldots \ldots \ldots \ldots \ldots \ldots \ldots \ldots \ldots \ldots \ldots \ldots$

3.2 Mechanical Properties of Sludge $\ldots \ldots \ldots \ldots \ldots \ldots \ldots \ldots$

3.3 Initial Finite Element Analysis $\ldots \ldots \ldots \ldots \ldots \ldots \ldots \ldots \ldots$

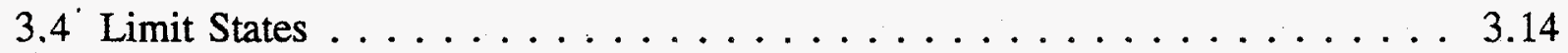

3.5 Finite Element Model . . . . . . . . . . . . . . . . . 3.15

3.6 Algorithm to Compute Limit States . . . . . . . . . . . . . . . 3.15

3.7 Random Variables $\ldots \ldots \ldots \ldots \ldots \ldots \ldots \ldots \ldots \ldots$

3.8 Sensitivity Measures $\ldots \ldots \ldots \ldots \ldots \ldots \ldots \ldots \ldots \ldots \ldots \ldots$

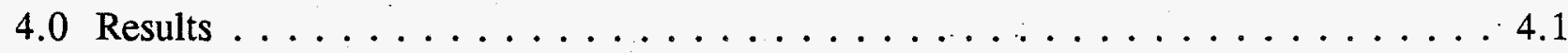

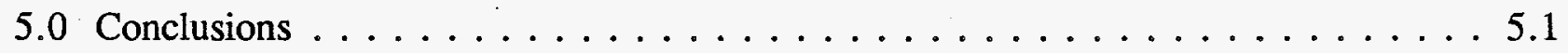

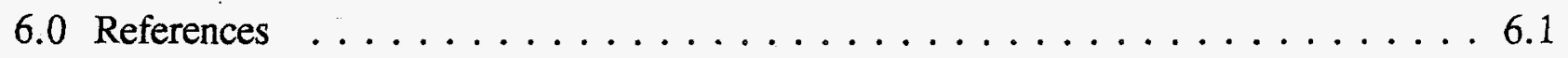





\section{Figures}

3.1 Two-Dimensional Finite Element Modeling of Solids of Revolution $\ldots \ldots \ldots$. . 3.2

3.2 Eight-Node, Axisymmetric Finite Element $\ldots \ldots \ldots \ldots \ldots \ldots . \ldots \ldots$

3.3 Deterministic Finite Element Model of Sludge $\ldots \ldots \ldots \ldots \ldots . \ldots \ldots$

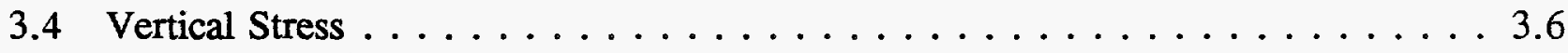

3.5 Displaced Shape of Sludge $\ldots \ldots \ldots \ldots \ldots \ldots \ldots \ldots \ldots \ldots$

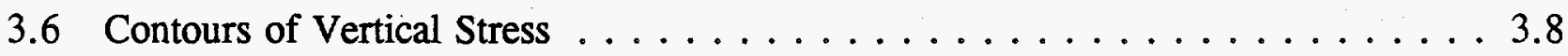

3.7 Contours of Radial Stress $\ldots \ldots \ldots \ldots \ldots \ldots \ldots \ldots \ldots$

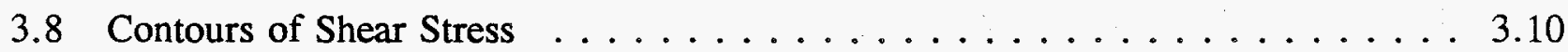

3.9 Vertical Stress at Bulge $\ldots \ldots \ldots \ldots \ldots \ldots \ldots \ldots \ldots \ldots \ldots \ldots \ldots$

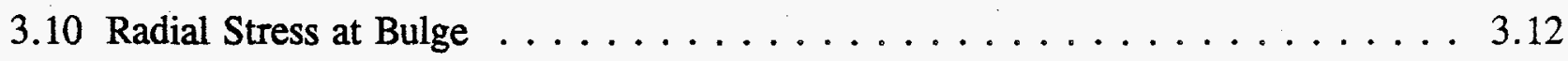

3.11 Shear Stress Near Bulge . . . . . . . . . . . . . . . 3.13

4.1 Probability of Failure Versus Time Step $\ldots \ldots \ldots \ldots \ldots \ldots \ldots .2$

4.2 Reliability Index Versus Time Step $\ldots \ldots \ldots \ldots \ldots \ldots \ldots . \ldots \ldots . . \ldots \ldots$

4.3 Void Percentage and Bubble Shapes for First Rollover Cycle for Tank $241-$ SY-101 . . . . . . . . . . . . . . . . . 4.3

4.4 Sensitivity of Reliability Index to Random Variables for Sludge with No Tensile Strength $\ldots \ldots \ldots \ldots \ldots \ldots \ldots . \ldots \ldots$

4.5 Sensitivity of Reliability Index to Random Variables for Sludge with Tensile Strength of $50 \mathrm{~Pa} \ldots \ldots \ldots \ldots \ldots \ldots . \ldots \ldots$

4.6 Sensitivity of Reliability Index to Random Variables for Sludge with Tensile Strength of $100 \mathrm{~Pa} \ldots \ldots \ldots \ldots . \ldots \ldots \ldots$

4.7 Vertical Stress Versus Height for Sludge with No Strength . . . . . . . . . . 4.6 
4.8 Vertical Stress Versus Height for Sludge with Tensile Strength of $50 \mathrm{~Pa} \ldots \ldots \ldots \ldots \ldots . \ldots \ldots$

4.9 Vertical Stress Versus Height for Sludge with Tensile Strength of $100 \mathrm{~Pa} \ldots \ldots \ldots \ldots . \ldots \ldots .7$

\section{Table}

3.1 Random Variables $\ldots \ldots \ldots \ldots \ldots \ldots \ldots \ldots \ldots \ldots . \ldots \ldots . \ldots \ldots$ 


\subsection{Introduction}

Stratification of the wastes in many of the Hanford storage tanks has resulted in sludge or slurry layers which are capable of retaining gases formed within the waste by chemical and/or radiolytic reactions. As the gas is produced, its pressure increases, and the mechanisms of gas storage evolve until a point of instability is reached. At that point, the sludge "rolls over" and a significant volume of gas is suddenly released. Because the releases may contain flammable gases, these major episodes are potentially hazardous. Mitigation techniques are desirable for more controlled releases of the gas at more frequent intervals.

To aid the mitigation efforts, a methodology for predicting the probability of sludge rollover at specific times is desired. This methodology would then provide a rational basis for developing a schedule for the mitigation procedures. That is, the build-up of gas could be released when the probability of a rollover event exceeds a certain predetermined value. In addition, a knowledge of the sensitivity of the sludge rollovers to various physical and chemical parameters within the tanks would provide direction for efforts to reduce the frequency of these events.

As part of the Flammable Gas Project at the Pacific Northwest Laboratory (PNL), ${ }^{(a)}$ researchers investigated the use of probabilistic finite element analysis for computing the probability of a rollover event. They also examined the sensitivity of such events to the various parameters.

The probabilistic study is described in this report. Background information on the mechanisms of gas bubble retention within the sludge and the resulting mechanical behavior is presented in Section 2. In Section 3, the capabilities of the probabilistic finite element software and its application to the analysis of the sludge at various stages of gas generation are discussed. Results of calculations are presented in Section 4, and conclusions are drawn in Section 5. References cited in this report are listed in Section 6.

(a) PNL is a multiprogram national laboratory operated for the U.S. Department of Energy under Contract DE-AC06-76RLO 1830 by Battelle Memorial Institute. 


\subsection{Background}

Gas bubbles retained in waste sludge have a variety of effects on sludge behavior. These effects are described in this section.

\subsection{Sludge Behavior}

A number of studies have been performed to quantify the sludge material behavior and the mechanisms of bubble generation for waste in double-shell waste storage tanks (Allemann et al. 1990a, 1990b, 1991, 1993; Trent and Michener 1993; Gauglitz et al. 1994, 1995). In these studies, the waste is reported to be separated into an upper convective layer of supernatant liquid and a lower nonconnective layer of sludge. The sludge consists of solid particles that are surrounded by the supernatant liquid. Within the sludge are bubbles of gas, which are continuously generated within the liquid.

Three basic types of bubble shapes have been reported (Gauglitz et al. 1994, 1995). In the first case, spherical bubbles are small enough to exist in the liquid which fills the pores of diameter $D_{p}$ between the solid particles. The growth of the bubbles at this stage is not affected by the weight or structure of the solid particles. As gas is generated, and the pressure within the bubbles grows, the diameter of the bubbles becomes equivalent to $D_{p}$. The bubbles then either lose their spherical shape and slip through the pores or retain a nearspherical shape and displace the sludge. The former bubble type, in which the bubbles finger through the pores, is called dendritic. It occurs when gravitational forces are dominant. The latter bubble type occurs when surface tension dominates. A "bond number" is the parameter that has been derived (Gauglitz et al. 1994; Gauglitz et al. 1995, Appendix $\mathrm{H})$ to distinguish between the two bubble types. The number is based on the ability of the bubble lift force to overcome both the weight of the sludge and its yield strength.

To predict the growth of the bubbles throughout the depth of the sludge and the resulting density values, a computer program entitled BOND6 was developed (Gauglitz et al. 1995, Appendix $\mathrm{H}$ ). The sludge simulation begins with the assumption that a rollover event has just occurred and that the sludge has just resettled. The initial bubble size distribution is computed on the basis of the hydrostatic pressure within the sludge pores, which is defined by the initial pressure at the surface of the supernatant $\left(\mathrm{P}_{0}\right)$, the void fraction of the supernatant at the surface $\left(\alpha_{0}\right)$, the mass density of the supernatant $\left(\rho_{1}\right)$, and the depth of the supernatant above the sludge $(\Delta)$. An iterative solution is used to determine if the bubbles are spherical or dendritic, and values of the void fraction, $\alpha$, are determined throughout the depth of sludge. Once the distribution of $\alpha$ is known, the net mass density of the sludge can be found. For spherical (or sludge-displacing) bubbles, the local sludge density, $\rho_{\mathrm{b}}$, is

$$
\rho_{b}=\left[(1-\phi) \rho_{s}+\phi \rho_{l}\right](1-\alpha)
$$


while, for dendritic bubbles,

$$
\rho_{b}=(1-\phi) \rho_{s}+(\phi-\alpha) \rho_{I}
$$

where $\phi$ is the pore volume and $\rho_{\mathrm{s}}$ is the mass density of the solids. Then, the net mass density is simply given as $\left(\rho_{\mathrm{b}}-\rho_{\mathrm{b}}\right)$.

After determining the initial distribution of bubbles, BOND6 models gas generation by increasing the gas pressure incrementally in a series of time steps. Because the gas generation rate is assumed to depend on temperature and the temperature is assumed to be a function of sludge depth, the gas generation is a function of depth (i.e., parabolic). In addition, the possibility of percolation is considered. Percolation is the tendency of bubbles to interconnect through the depth of sludge. In this way, some of the gas is allowed to shift when the void fraction exceeds a percolation threshold. At the top of the sludge, the excess gas is assumed to be released into the supernatant. At points below, the gas generation rate is revised to reflect percolation, but the excess gas is assumed to remain at that level. On the basis of the gas generation and percolation rates, a new value of $\alpha$ and, subsequently, net density can be computed at any depth within the sludge.

For the BOND6 program, a rollover criterion was devised in which, for each time step (which is actually a gas generation step), the net mass density is computed at each depth within the sludge. A rollover event is assumed to occur when the net mass density becomes negative (i.e., buoyant) at any point.

According to Gauglitz et al. (1995, Appendix H), BOND6 performed well in modeling the sludge behavior of Tank 241-SY-101 as observed before December 1991. However, several limitations are possibly significant. The first is that the rollover criterion does not include any effects of the strength of the sludge material. Although an attempt was made to model the sludge above the point of buoyancy as a series of beams, the approach in BOND6 is simplistic. In addition, the approach is based on a one-dimensional grid through the depth of the sludge and thus cannot account for any horizontal variations of either sludge properties or bubble generation. Finally, it should be noted that the actual in situ properties of the sludge are known only approximately and that the value of the solution is therefore subject to their variance.

\subsection{Structural Reliability Analysis Program}

The CALREL (CAL-RELiability) general-purpose structural reliability program (Liu et al. 1989; Liu and Der Kiureghian 1989) was used for the reliability analyses that are described in this report. The structural reliability analysis methods employ concepts of probability and statistics to account for the uncertain nature of both the structures themselves and their loading environment. Probabilistic (or stochastic) methods of structural analysis 
were conceived in the 1970 s, and one of the fundamental techniques is the so-called reliability method.

The objective of reliability methods is to establish the probability of failure of a structure. There are two basic assumptions. First, all uncertainties are modeled with random variables. These random variables could represent such items as material properties, geometry of the structure, or applied loads. The second assumption is that the structure may fail in any of a finite number of modes and that, for any combination of values of random variables, the structure is either in a safe state or a failure state with respect to each mode. The failed or unfailed state of the structure for each mode is determined by the value of a limit-state function.

Limit-state functions for structures are usually defined on the basis of some aspect of the structural response. For example, failure might be defined in terms of the maximum stress at some point, maximum displacement, or instability. The vector of basic random variables is denoted as $\mathbf{V}$. For a set of the response threshold (or limit) values (such as material strength or a displacement limit), a vector of the structural response (such as stress or displacement when subjected to load) is denoted as $\mathbf{S}$ and a limit-state function, $g(\mathbf{V}, \mathbf{S})$, is defined such that $g(\mathbf{V}, \mathbf{S}) \geq 0$ defines a safe state and $g(\mathbf{V}, \mathbf{S}) \leq 0$ defines a failure state. The boundary between the two states, denoted by $g(\mathbf{V}, \mathbf{S})=0$, is called the limit-state surface. The probability of failure for any failure mode is given as

$$
P_{f}=\int_{g(V, S) \leq 0} f_{v}(V) d v
$$

where $f_{v}(V)$ is the joint probability density function of $V$. If only one limit state is considered, the evaluation is called a component reliability analysis. If several failure modes are included, a system reliability analysis is required.

For the solutions described in this report, the First Order Reliability Method (FORM) was used to solve Equation (3). With this method, the vector of random variables, V, is transformed into a vector of statistically independent standard normal variates, $\mathrm{Y}=\mathrm{Y}(\mathrm{V})$. This transformation is called the probability transformation, and it is invertible. Details are given by Liu and Der Kiureghian (1989).

Each limit-state function may now be equivalently defined as

$$
G(Y)=g(V(Y), S(V(Y)))
$$

and, in the space of the independent standard normal variates, $Y$, the probability of failure may be expressed as 


$$
P_{f}=\int_{G(Y) \leq 0} \phi(y) d y
$$

where $\phi(y)$ is the standard normal density of $\mathrm{Y}$.

In the standard normal space, the value of the integral of Equation (5) is affected most by the contribution from the region around the point on the limit-state surface nearest to the origin. Therefore, an approximation to the integral may be obtained by replacing the actual limit-state surface at that point with an approximate surface. With FORM, a tangent hyperplane is used. Then, the point nearest the origin, denoted $y^{*}$, is called the design point; one may think of it as the combination of values of random variables that is most likely to result in failure. The distance from $y^{*}$ to the origin, denoted $b$, is the reliability index.

The effective use of FORM requires that the design point, $\mathrm{y}^{*}$, be found efficiently. This is a constrained minimization problem (i.e., the distance to the origin is minimized, subject to the constraint that the final point must lie on the limit-state surface), and a number of mathematical algorithms have been proposed to solve it. For the work documented in this report, the Sequential Quadratic Method was employed.

If multiple limit-state functions are required, then each has its own design point, reliability, and probability of failure. If system failure occurs with the satisfaction of any limit-state function independently, it is called a series system and it would be analogous to a statically determinate structure. Failure would then be controlled by the limit state with the highest probability of failure. However, if all of the limit-state functions must be satisfied for failure, it is a parallel system; directional simulation in conjunction with FORM is required to obtain overall system properties.

In addition to values of probability of failure and reliability, CALREL has the capability to compute a sensitivity factor of the solution with respect to each random variable. That is, for any random variable, $\mathrm{q}$, the sensitivity factor with respect to the reliability index, $\mathrm{b}$, is simply the derivative $\mathrm{db} / \mathrm{dq}$. Four types of sensitivity factors are available:

- The parameter, $\mathrm{a}$, is the sensitivity of $\mathrm{b}$ with respect to the design point in standard normal space.

- Parameter $\mathrm{g}$ is the scaled and normalized sensitivity of $\mathrm{b}$ with respect to the design point in original space.

- Parameter $d$ is the scaled sensitivity of $b$ with respect to the mean of each random variable. 
- Parameter $h$ is the scaled sensitivity of $b$ with respect to the standard deviation of each random variable. 


\subsection{Solution Procedure}

The CALREL software was written to perform probabilistic analyses in which, given a set of limit-state functions and random variable information, the design point is found on the basis of a minimization algorithm, leading to a definition of the probability of failure, the reliability index, and the sensitivities of the solution with respect to each random variable. However, the user must supply a fairly extensive program segment that is able to supply, for given values of the random variables, an evaluation of the limit-state function. To obtain the stress state within the sludge material, a finite element analysis routine was a required part of the user-supplied program.

\subsection{Finite Element Software}

From observations of sludge behavior (Gauglitz et al. 1994), the discrete release of gas bubbles occurs when the sludge fails mechanically (i.e., it yields and begins to flow). A bubble will either rise through the sludge or it will cause the sludge to rise in gobs. Therefore, for round bubbles away from the edge of the tank, an axisymmetric analysis is most appropriate.

Axisymmetric models are two-dimensional representations of solids of revolution. They are based upon a cylindrical coordinate system, $r, z$, and $\theta$, as shown in Figure 3.1. All geometry, material properties, and loads are assumed to be independent of $\theta$. Each node is considered to represent a circle with two degrees of freedom consisting of $\mathrm{u}$ (radial) and $\mathrm{w}$ (vertical) displacements. Stress components include radial stress, $\sigma_{\mathrm{r}}$; vertical stress, $\sigma_{z}$; hoop stress, $\sigma_{\theta}$; and shear stress, $\tau_{z x}$. Small deflections/deformations and a linear elastic material were assumed. Derivations and descriptions of axisymmetric finite elements may be found in standard textbooks (e.g., Cook et al. 1989).

A finite element module was written specifically for this application. Eight-node isoparametric finite elements were implemented, as shown in Figure 3.2. Elements of this type are derived on the basis of quadratic shape functions and are, therefore, capable of modeling domains with curved sides. Gauss quadrature was used with a grid of $3 \times 3$ integration points, which is considered to be full integration (Cook et al. 1989). Values of the stress components are evaluated at four points within each element, corresponding to $2 \mathrm{x}$ 2 integration points for accuracy of shear stress.

Loading in the finite element model is provided by the net weight density as a body force. Although weight density is considered as a material property in most finite element analyses, here it is defined as a nodal value so that the variation of weight density spatially and with depth of sludge can be modeled. 

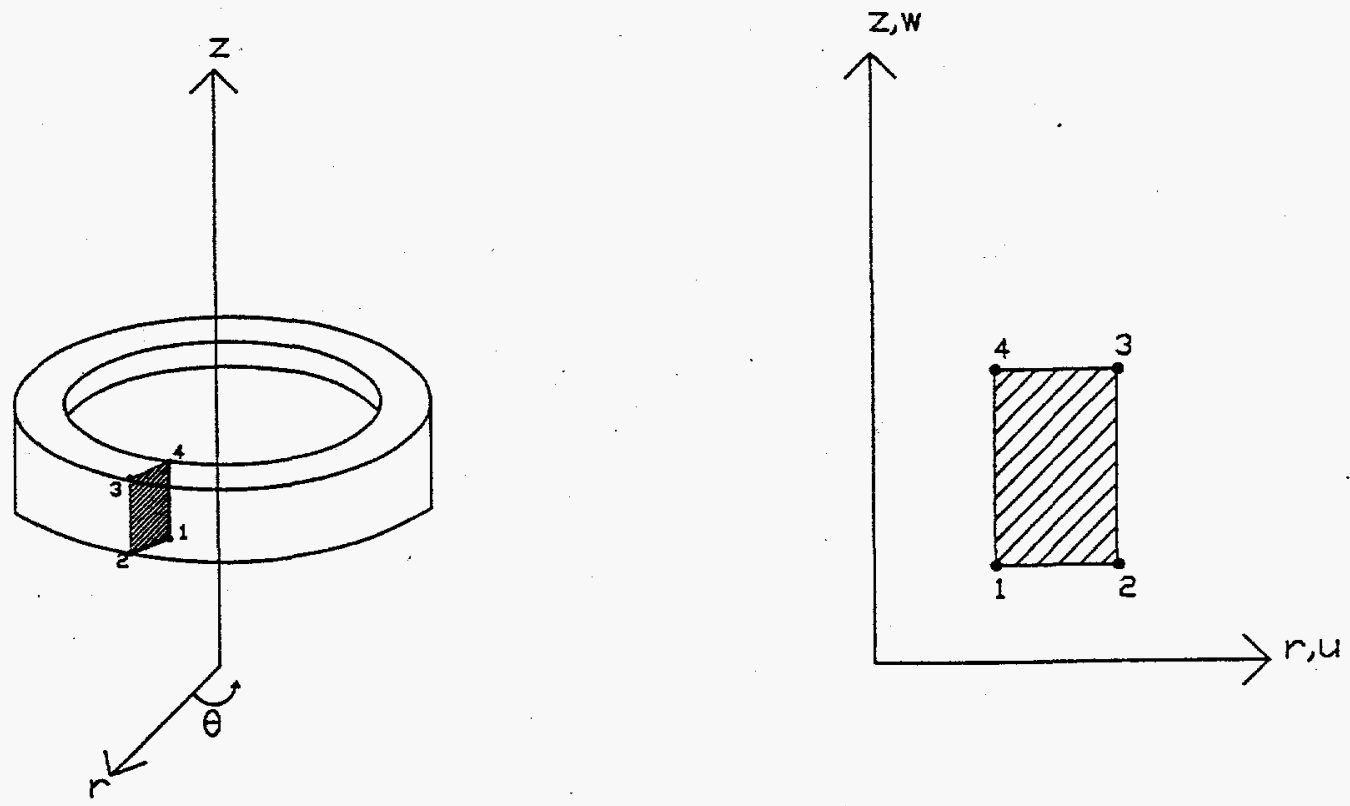

Figure 3.1. Two-Dimensional Finite Element Modeling of Solids of Revolution

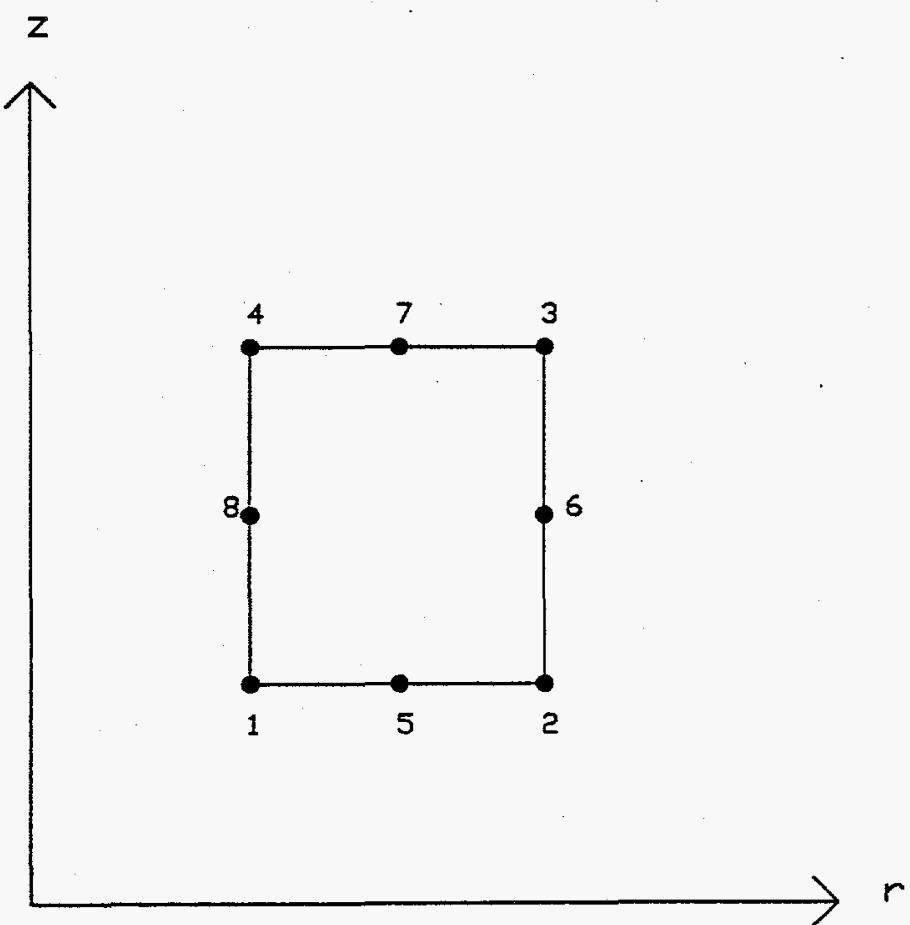

Figure 3.2. Eight-Node Axisymmetric Finite Element 


\subsection{Mechanical Properties of Sludge}

The sludge is thought to be a thick, cohesive, semisolid with very low (but nonzero) shear and tensile strength values. ${ }^{(a)}$ For the work reported here, the sludge material is modeled as a solid material that remains elastic until yield or tensile failure occurs, at which point elastic assumptions are no longer valid. On the basis of previous work, ${ }^{(b)}$ the bulk modulus, $\mathrm{k}$, was assumed to be $2 \mathrm{GPa}$; Poisson's ratio, $\nu$, was assumed to be 0.45 to reflect a nearly incompressible material. Thus, the modulus of elasticity, $\mathrm{E}$, is $0.6 \mathrm{GPa}$, and the shear modulus, G, is $0.2 \mathrm{GPa}$.

Other properties were provided by Gauglitz et al. (1995, Appendix H):

Depth of supernatant, $\Delta: 4.57 \mathrm{~m}$

Supernatant density, $\rho_{1}: 1503 \mathrm{~kg} / \mathrm{m}^{3}$

Surface tension: $0.065 \mathrm{~N} / \mathrm{m}$

Sludge depth: $5.26 \mathrm{~m}$

Solids density, $\rho_{\mathrm{s}}: 2100 \mathrm{~kg} / \mathrm{m}^{3}$

Pore volume, $\phi: 0.686$

Pore diameter $\mathrm{D}_{\mathrm{p}}: 3 \times 10^{-6} \mathrm{~m}$

Percolation threshold: 0.15 for round bubbles

0.10 for dendritic bubbles

Initial void fraction, $\alpha_{0}: 0.122$

Initial bubble diameter, $D_{0}: 1 \times 10^{-4} \mathrm{~m}$

Very little is known about the strength properties of the sludge. According to an unpublished source, the sludge shear strength depends significantly on mixing, dilution, and heating (Tingey et al. 1994). Reported values include 12,000 Pa, $265 \mathrm{~Pa}, 200 \mathrm{~Pa}, 70 \mathrm{~Pa}$, and $65 \mathrm{~Pa}$ (Gauglitz et al. 1995, Appendix $\mathrm{H}$ ); $600 \mathrm{~Pa}$; ${ }^{(b)}$ and $60 \mathrm{~Pa}, 20 \mathrm{~Pa}$, and $0.6 \mathrm{~Pa}$ (Tingey et al. 1994). These values span nearly two orders of magnitude. According to Gauglitz et al. (1994), the shear strength and tensile strength are related by a proportionality constant, but a stress failure theory has not been proposed.

\subsection{Initial Finite Element Analysis}

To investigate the behavior of the sludge at incipient rollover and to determine the corresponding state of stress, a deterministic finite element analysis was performed. An

(a) Johnson, K. I., and J. D. Hudson. 1994. Feasibility of Applying the ANSYS Finite Element Code to the Simulation of Incipient Sludge Turnover. Letter Report PNLFGP: 05.27.94, Pacific Northwest Laboratory, Richland, Washington.

(b) These values were provided by K. I. Johnson in a Flammable Gas Project quarterly review meeting, 1995. 
axisymmetric model (Figure 3.3) of the sludge in Tank 241-SY-101 was generated. The aforementioned elastic properties were assumed for a linear analysis, and the results of the BOND6 program for the first rollover step were used to compute the net weight density for any level of sludge, as depicted in Figure 3.3 by shading. The center of the tank corresponds to the leftmost column of nodes. Note that the bottom and sides of the tank were assumed to be "smooth" in that the sludge was constrained only in the direction perpendicular to the tank wall.

As previously mentioned, the BOND6 program is based on a one-dimensional vertical grid in which no horizontal spatial variation is allowed. An initial analysis was performed in which all properties and loads were assumed to be uniform in the radial direction. As expected, the response was also uniform. A plot of the vertical stress (denoted as sigma y) versus depth of sludge is shown in Figure 3.4, where stress is given in pascals. Distance on the horizontal axis refers to depth within the sludge, for which values of 0 and 1 correspond to the top and bottom surfaces, respectively. The region of buoyant sludge is indicated by a positive slope of the curve, leading to a reduction in compressive vertical stress with depth. The most tensile value occurs at the bottom of the buoyant region. Here, the stress is slightly tensile, verifying that this is the step in which rollover would occur, as indicated by the BOND6 output.

For shear stresses to occur in the sludge, the sludge density must vary in the radial direction. Thus, another analysis was performed in which the overall weight density of the sludge material was reduced by $10 \%$ for the leftmost three columns of elements, corresponding to the center of the axisymmetric region. An exaggerated plot of the displaced shape of the mesh is given in Figure 3.5, showing the bulge that results. Contour plots of vertical stress, radial stress, and shear stress are shown in Figures 3.6, 3.7, and 3.8, respectively. In Figures 3.6 and 3.7, the vertical and radial stress is shown to vary slightly in the region of decreased density. A plot similar to that of Figure 3.4 is given in Figure 3.9 , in which one may note that the vertical tensile stress has nearly doubled. A plot of radial stress (denoted as sigma $x$ ) near the center of the bulge is shown in Figure 3.10. Because of near-incompressibility, the plot is very similar in shape to that of vertical stress. However, one should note that the maximum tensile stress occurs at the top of the bulge. From Figure 3.8, one can see that, aside from anomalous stress that occurred at the boundaries, all shear was concentrated at the edge of the bulge. A plot of the variation of shear stress with depth is presented in Figure 3.11. Again, stress is given in pascals. One should note that the maximum values of all stress components are of the same order of magnitude. 


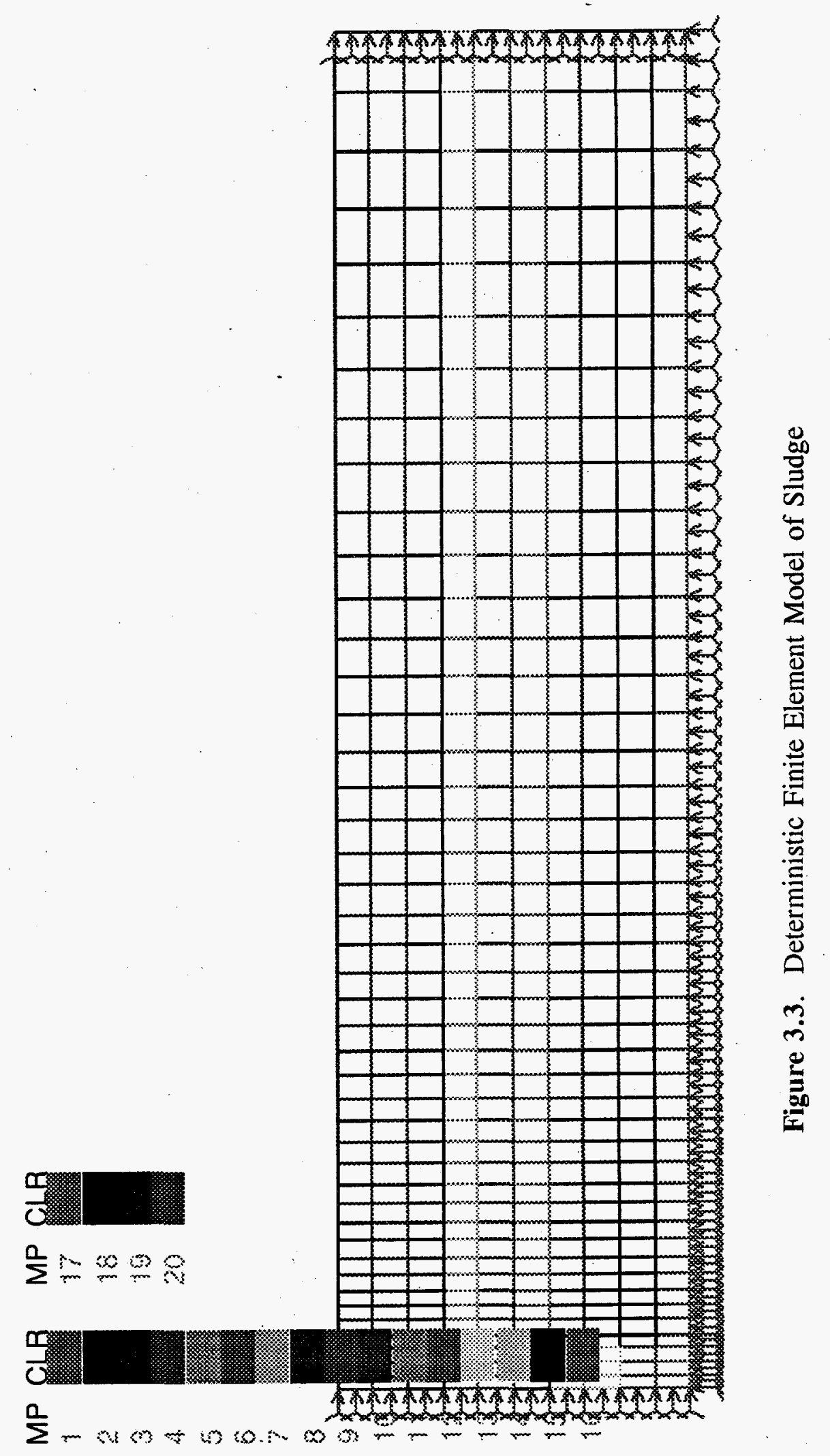




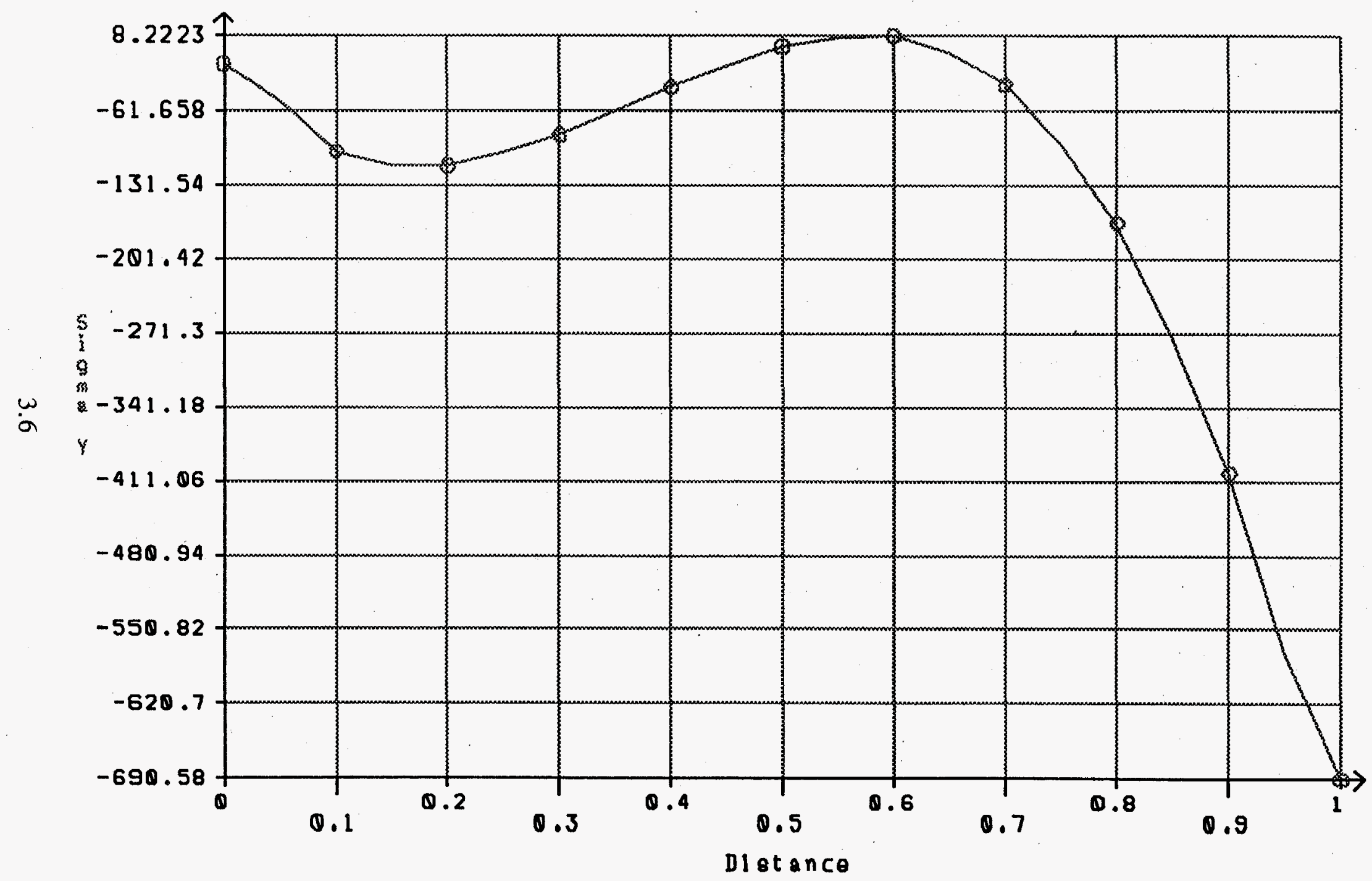

Figure 3.4. Vertical Stress 


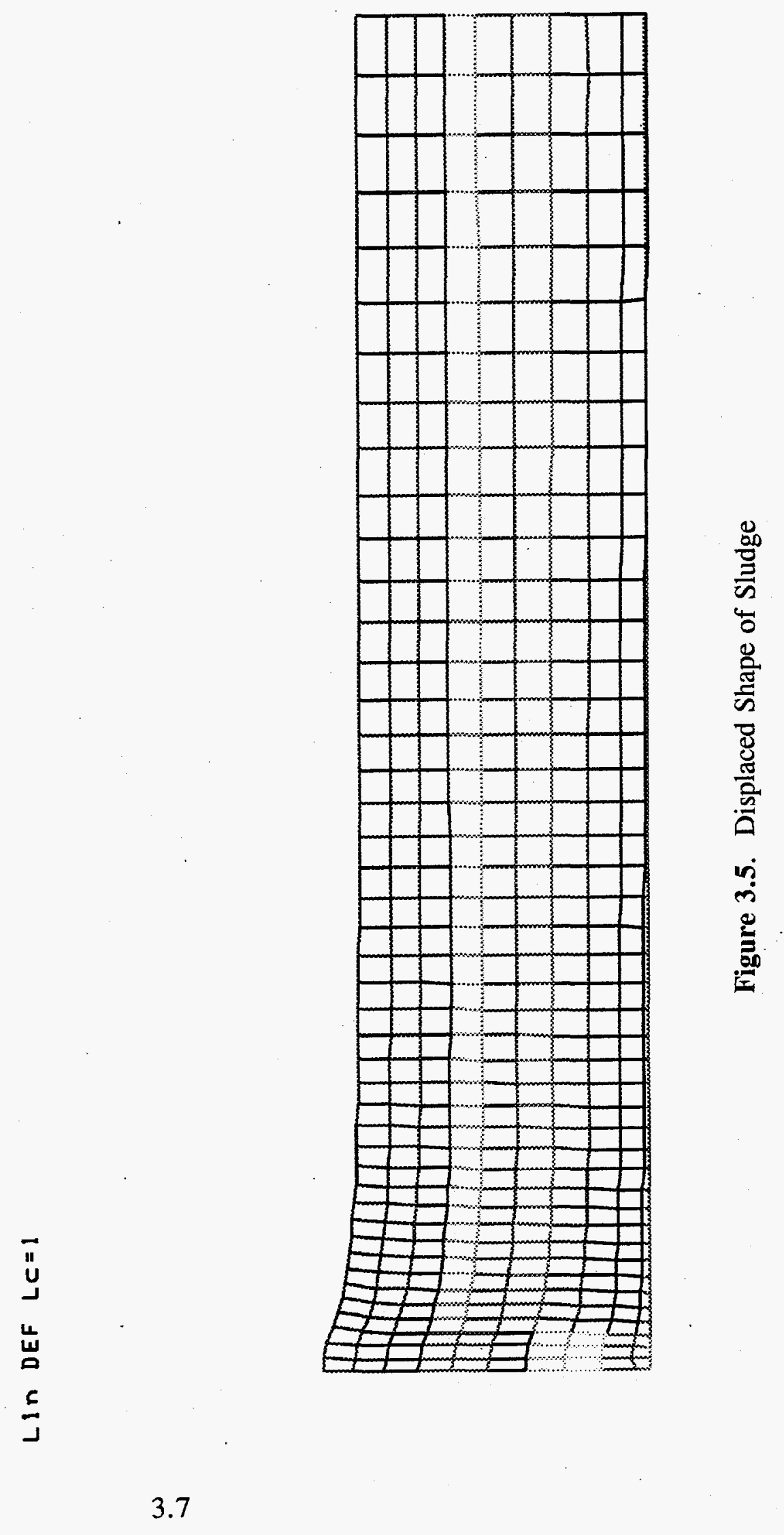


Lin STRESS LC $=1$

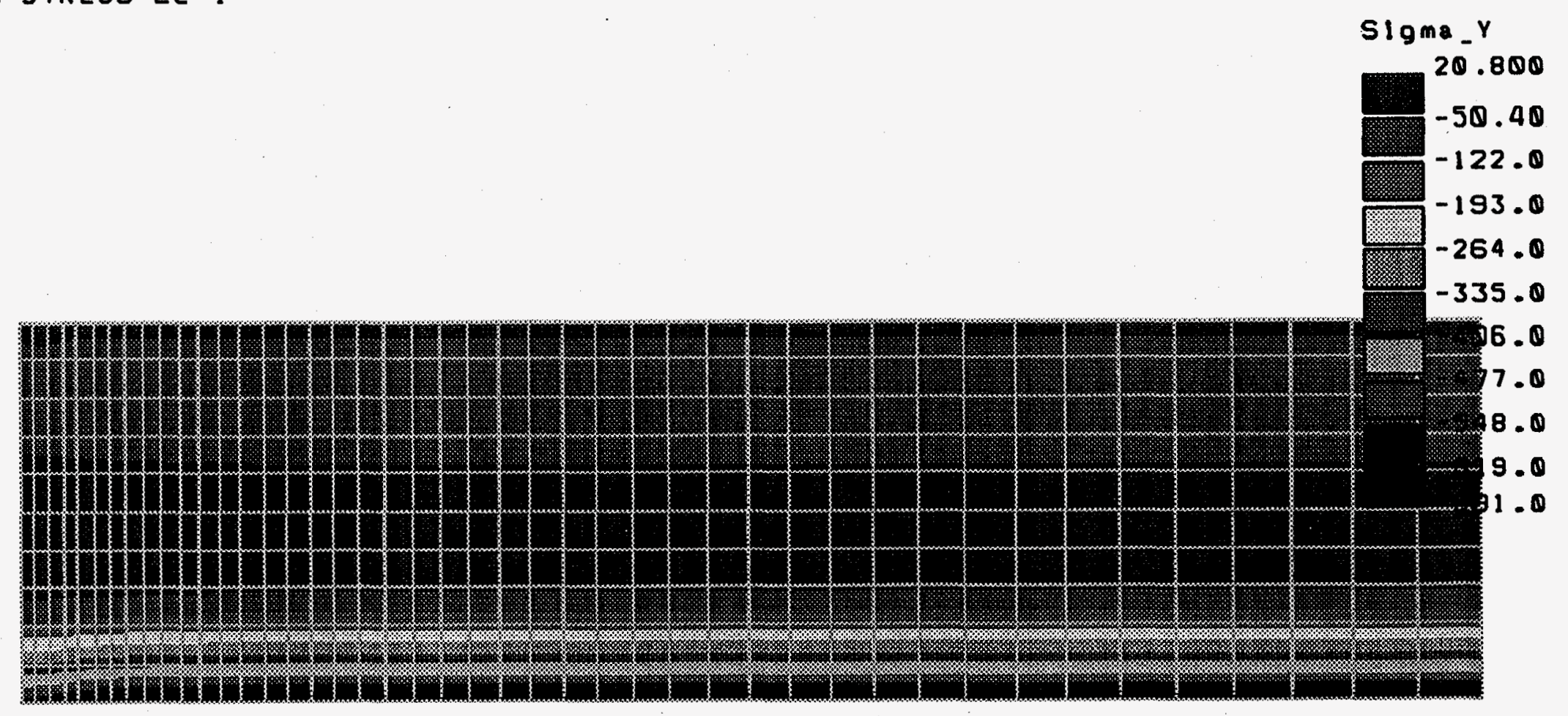

Figure 3.6. Contours of Vertical Stress 


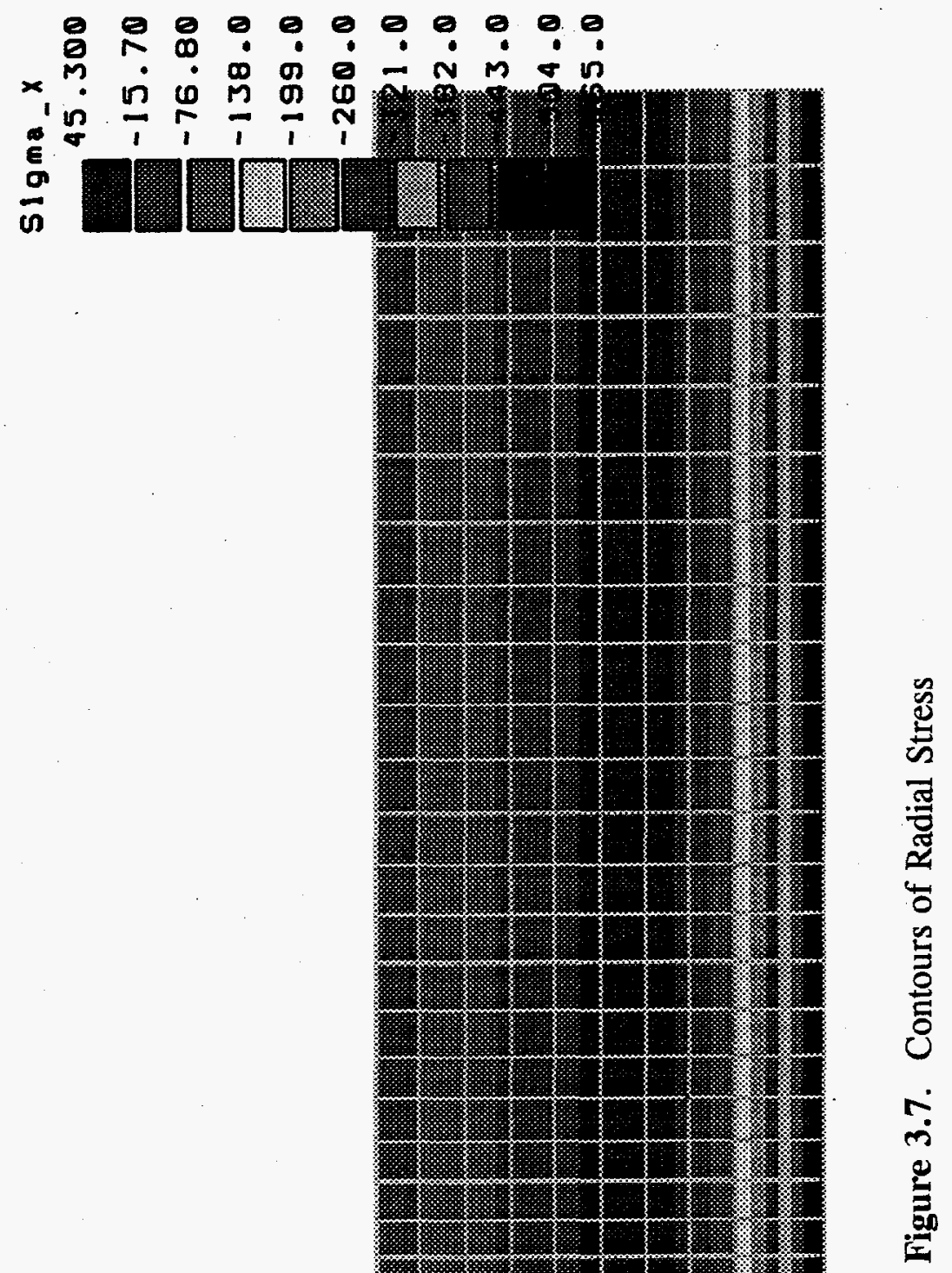

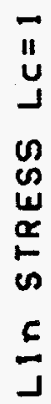

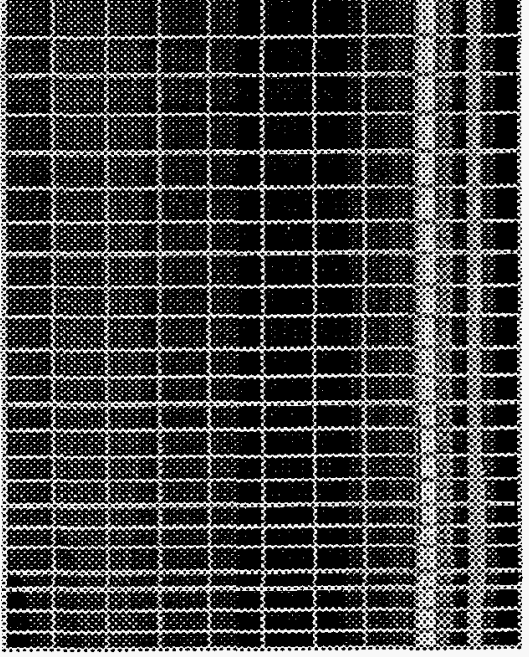




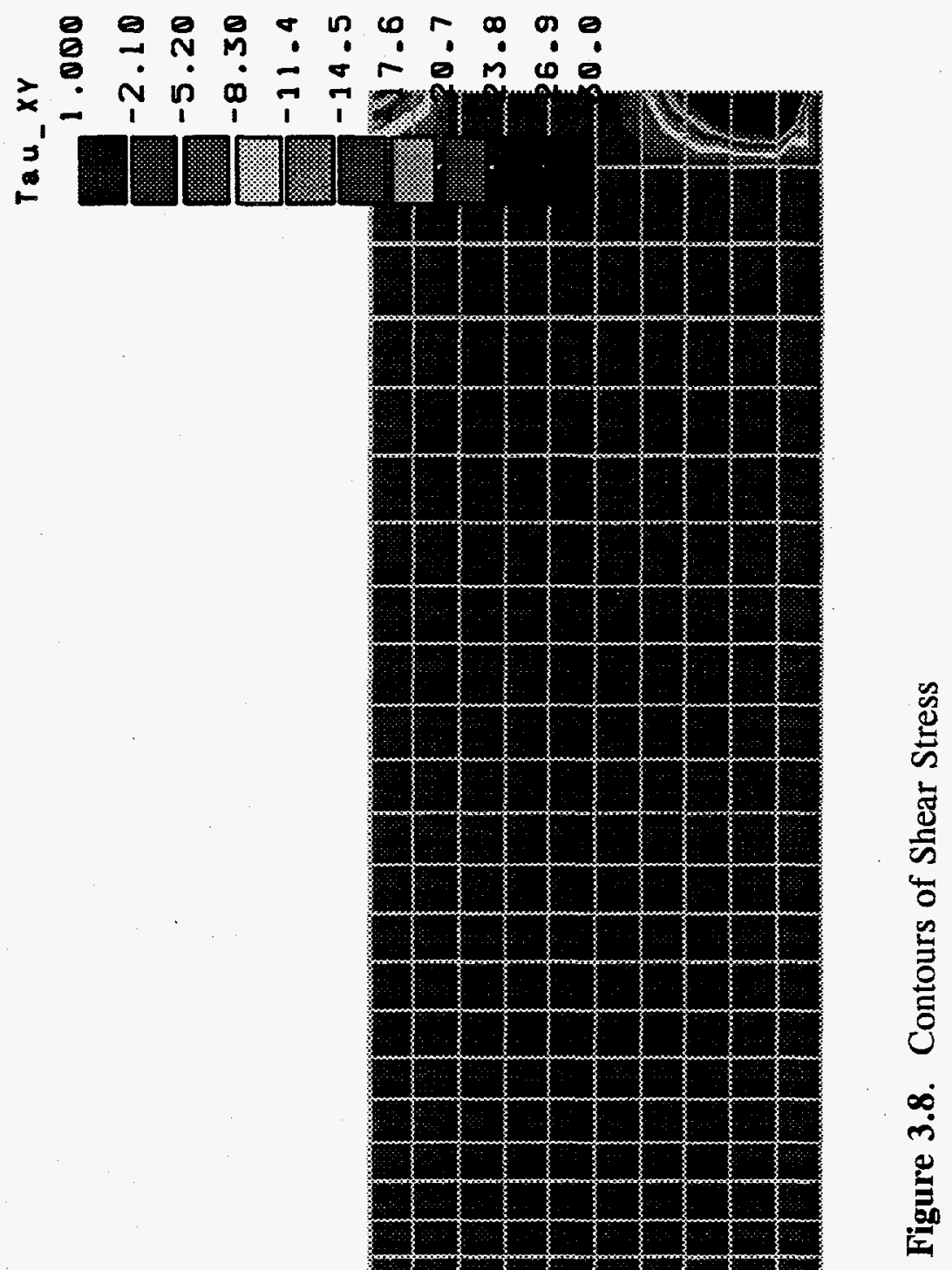

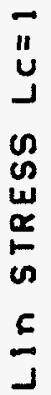

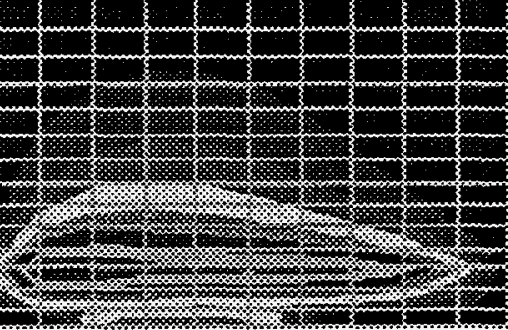

3.10 


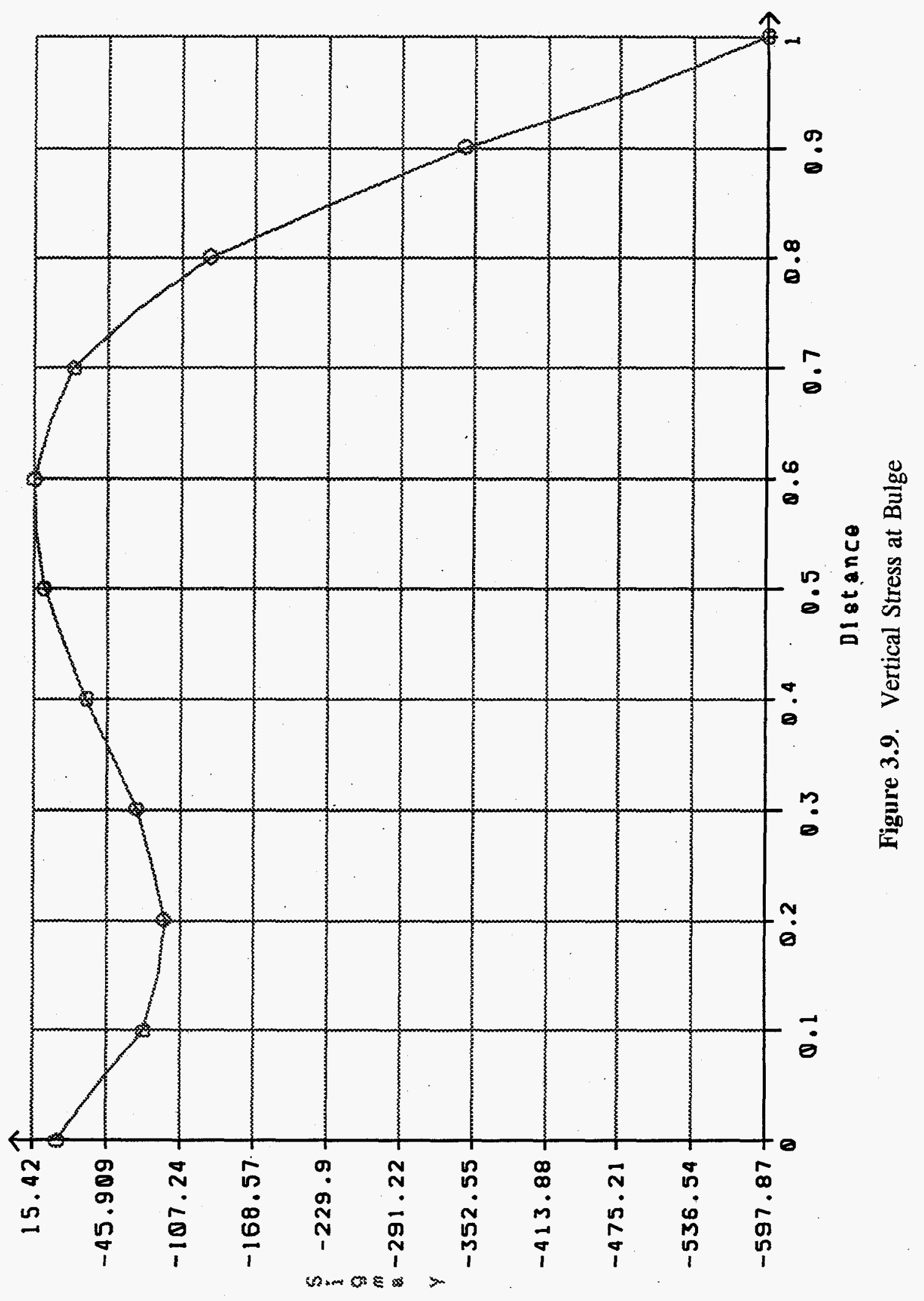

3.11 


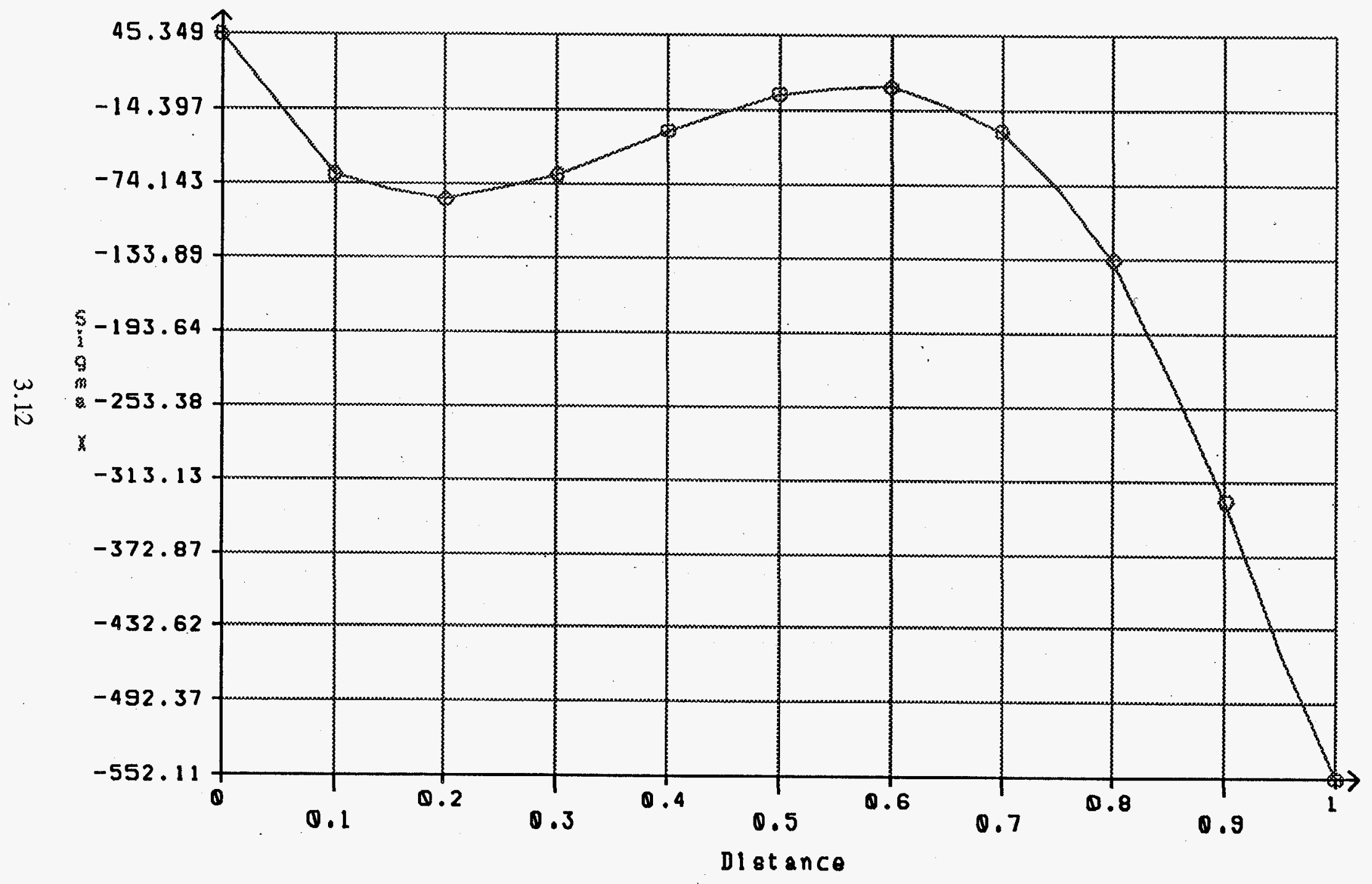

Figure 3.10. Radial Stress at Bulge 


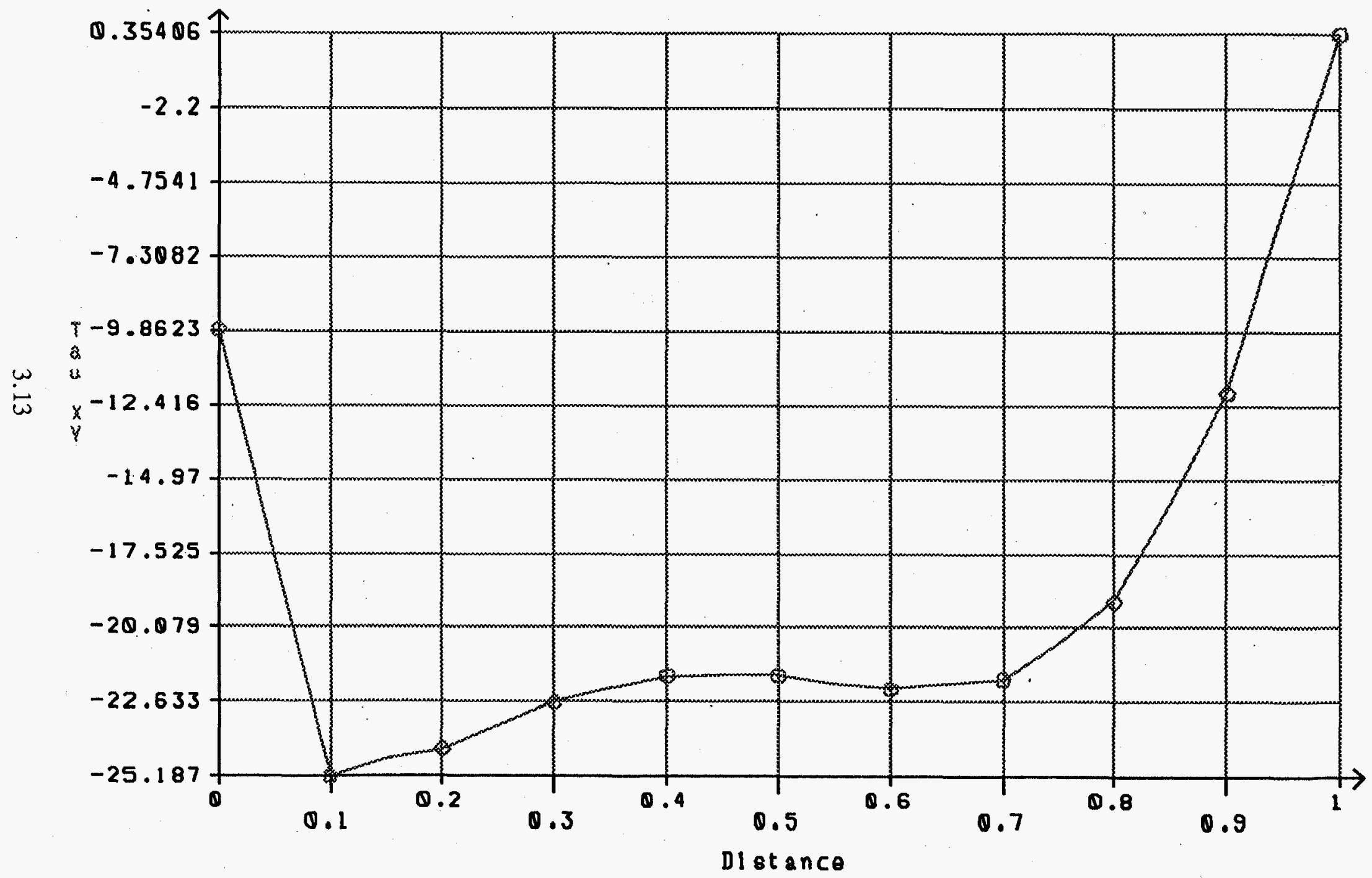

Figure 3.11. Shear Stress Near Bulge 


\subsection{Limit States}

To perform probabilistic finite element analysis, the definition of limit states must be specified. As mentioned earlier, failure is typically defined by comparing aspects of the structural response, such as stress or displacement, to allowable values. The simplest type of analysis is component reliability analysis, in which only a single limit state is considered. Thus, a single stress measure that could be monitored for the entire model was desired to indicate failure.

The first limit state that was considered is analogous to that used by Gauglitz et al. (1995, Appendix H) in the BOND6 program, for which the sludge is assumed to have no strength. That is, rollover is assumed to occur when the buoyancy of some portion of the sludge is large enough to overcome the weight of the sludge above. This state is indicated when the vertical stress, $\sigma_{z}$, below the buoyant portion becomes positive (i.e., tensile). Therefore, this limit state may be defined as

$$
g(V, S)=-\bar{\sigma}_{z, \max }
$$

where $\sigma_{\mathrm{z}, \max }$ is the maximum vertical stress for the entire domain of the sludge model. For this limit state, failure is thus defined when some point within the model attains zero vertical stress.

For the second limit state, the effect of the strength of the sludge material is included. A number of stress-based failure theories have been used for structural materials. For brittle materials, one might consider the maximum principal stress, while for ductile materials, the maximum shear stress or maximum von Mises stress would be most appropriate. Intuitively, for the buoyant portion of the sludge to flow, both the sludge in tension below and above the buoyant region and the sludge subjected to shear along the side must yield.

Several preliminary CALREL analyses were performed to determine the most practical failure criterion. When the maximum von Mises stress failure criterion was chosen, the resulting failure points were determined by high compressive stress at the bottom of the tank, which is not indicative of a rollover event. When the maximum shear stress failure criterion was chosen, the lack of significant spatial variation in buoyancy and the corresponding small values of shear stress resulted in failure points with unreasonably large values of vertical tensile stress. Again, the failure points did not correspond to a rollover event, as observed from the deterministic analysis. Finally, a maximum vertical stress failure criterion, similar in concept to the first limit state, was applied. Satisfactory results were obtained. In this case, failure is assumed to occur when the maximum vertical tensile stress reaches a limiting value. The limit-state function is

$$
g(V, S)=\sigma_{\text {lim }}-\sigma_{z}
$$


where $\sigma_{\lim }$ is the limiting value of tensile stress. Because of the wide range of reported values and the uncertainty of the failure process, two levels of sludge tensile strength were considered, varying from $50 \mathrm{~Pa}$ to $100 \mathrm{~Pa}$.

One may note that, although the second limit-state function includes the strength of the sludge up to initial yield in tension, the actual flow of the buoyant portion will not occur until all tensile and shear areas of the sludge have yielded. The yield of one portion will certainly affect the subsequent behavior of the other portions and, if it is desired to model yield and flow, a nonlinear analysis capability is required, which is beyond the scope of this report.

\subsection{Finite Element Model}

The axisymmetric finite element model of the sludge of Tank 241-SY-101 was shown in Figure 3.3. A domain with radius $2.5 \mathrm{~m}$ is considered to be a representative volume of the material, and the buoyant region is assumed to be centered at the axis of symmetry.

Rectangular elements were used to avoid distortion effects, and the aspect ratio of the elements was kept below 4 . The mesh was kept fairly fine in the $r$-direction to minimize adverse effects at the center, and the nodes were equally spaced to simplify modeling of the spatial variation of the random variables. Nodes along the bottom surface were constrained in the vertical direction, while nodes on the outer radius and at the center were constrained in the radial direction.

\subsection{Algorithm to Compute Limit States}

As previously mentioned, a user-defined program segment was required to define the value of the limit-state function for any set of values of the random variables. This program segment had two main components: 1) significant portions of the BOND6 program to compute the net weight density of sludge as a function of depth and 2) the aforementioned finite element program.

Values for the random variables are provided by the CALREL program, while all other input data is read from a separate file. Basically, for each column of nodes in the finite element mesh, the procedure from BOND6 is used to compute the net mass density of the sludge that exists after a certain number of user-defined time steps. These nodal values of mass density are converted to weight density (negative values are buoyant) and passed to the finite element subroutines via a scratch file. After values are defined for all nodes, the stress analysis is performed and the stress solution (four sets of components per element) is used to compute the value of the limit-state function. The algorithm is as follows:

1. Loop through the columns of nodes, each of which has been assigned a value for $\alpha_{0}$. 
2. For this column, call BOND6 subroutines to obtain values of net mass density through the depth. Assign values to each node of this column.

3. End loop.

4. Call the finite element subroutines with the given values of net density. Compute nodal displacements and element stresses.

5. Loop through all elements within a certain region of the mesh. Here, elements around the outer edge and, often, the top layer are ignored because of possible anomalies in stress.

6. Check for the maximum value of $\sigma_{\mathrm{z}}$.

7. End loop.

8. Define the limit-state function.

9. Return.

\subsection{Random Variables}

Five variables were chosen to be random. The values assigned to these variables were as described for the BOND6 program (Gauglitz et al. 1995, Appendix H), but with an estimated normal statistical distribution. The mean values and standard deviations given in Table 3.1. One should note that a correlation matrix was provided for values of the initial void fraction, $\alpha_{0}$, which are assigned for each vertical column of nodes. Although the actual statistical distributions for the random variables could not be established, the values that were chosen represent reasonable estimates based on discussions with PNL staff involved in the characterization and modeling of sludges in Hanford tanks. As statistical data become available, the distributions may be revised.

Table 3.1. Random Variables

\begin{tabular}{lcccc}
\multicolumn{1}{c}{ Random Variable } & & $\begin{array}{c}\text { Mean } \\
\text { Value }\end{array}$ & & $\begin{array}{c}\text { Standard } \\
\text { Deviation }\end{array}$ \\
\cline { 1 - 1 } Initial Void Fraction, $\alpha_{0}$ & & 0.122 & & 0.0122 \\
Density of Supernatant, $\rho_{1}$ & & 1503 & & 150.3 \\
Depth of Supernatant, $\Delta$ & & 4.57 & & 0.229 \\
Reference Pressure, $\mathrm{P}_{0}$ & & 101,000 & & 10,100
\end{tabular}




\subsection{Sensitivity Measures}

A sensitivity measure in this study reflects both the physical and stochastic importance of a parameter (e.g., pressure, supernatant density). The potential uses of the sensitivity measures are to identify needs for 1) obtaining more data and 2) controlling the most important parameter. In general, the values of the sensitivity measures depend on

- the mean, standard deviation, and type of distribution of each random variable

- the interaction between all of the random variables

- the value of the reliability index (e.g., a high reliability index normally results in lower sensitivity measures)

- the nature of the limit state function and its dependence on certain random variables.

The sensitivity factors (i.e., alpha values) are the directional cosines in the uncorrelated normalized space of the random variables. They are evaluated using the reliability code. A variable with a large absolute value of alpha is considered to be stochastically and physically important. The stochastic importance depends on the variabilities of the parameters and the value of the reliability index, while the physical importance is related mainly to the nature of the failure function. Furthermore, the sum of the squares of the sensitivity measures (i.e., alpha values) is unity. In addition, it has been observed that alpha values reflect the dependency of the failure probability (or the reliability index) on a small variation of the mean value of a variable. 


\subsection{Results}

The sludge in Tank 241-SY-101 was analyzed for each of the first 15 time steps selected by Gauglitz et al. (1995, Appendix H). According to Gauglitz et al., the model data corresponded to the conditions in the tank in the time period of August to December 1991, and each time step was said to equal approximately 8 days. As previously mentioned, three estimates of sludge strength were considered: no strength, a tensile strength of $50 \mathrm{~Pa}$, and a tensile strength of $100 \mathrm{~Pa}$. One may note that convergence in the minimization algorithm was not obtained for time steps 4 and 8 when the tensile strength was set to $50 \mathrm{~Pa}$ and time steps 5 and 8 when the tensile strength was set to $100 \mathrm{~Pa}$. Plots of the probability of failure versus time step for each case are given in Figure 4.1. Similar plots of the reliability index are given in Figure 4.2.

As expected, the probability of failure grows and the reliability index diminishes with increasing time. Also, the reliability increases for all time steps with increased sludge strength, although the effect is greatest for the early steps and the last step. Note that the reliability index has the appropriate zero value when the probability of failure is $50 \%$.

The curves seem to exhibit two nearly linear regions. The first includes steps 1 through 4 , while the second is from step 4 to 14 . As a point of comparison, the deterministic analysis results from work on the BOND6 program are reproduced in Figure 4.3 (Gauglitz et al. 1995, Appendix H). From steps 1 to 4 , the void volume percentage appears to increase monotonically from the top of the sludge to the bottom, with no internal pockets of buoyancy. A rollover event would likely occur near the top of the sludge, with minimal gas release. At step 4, however, a region of heightened void volume within the sludgedisplacing region begins to appear, leading to a change in the rate of increase of probability of failure. For the next ten steps, the probability of rollover increases at a nearly constant rate as gas is generated. The analysis predicted a rollover event at step 14, at which point the reliability analysis for the case of no sludge strength indicates a dramatic increase in the probability of failure (Gauglitz et al. 1995, Appendix H).

Bar graphs showing the sensitivity of the solutions to the random variables are given in Figures 4.4 through 4.6. From these graphs, one may note that, for a large majority of the cases, the reliability is most sensitive to the value of supernatant density. This result is not surprising because the net density and, hence, the buoyancy, are directly related to the density of the liquid. The graphs in Figures 4.5 and 4.6 are somewhat different from that of Figure 4.4 due to the addition of sludge strength. In the early stages of bubble growth (time steps 1 through 4), the value of void fraction at the surface of the supernatant, $\alpha_{0}$, is relatively significant when the sludge is assumed to have no strength. Also, when the sludge has no strength, a radical change occurs for failure at step 15 , at which time the supernatant density loses significance and pressure in the tank becomes important. From these graphs, it is apparent that sludge strength tends to stabilize the failure (i.e., rollover) mechanism. 


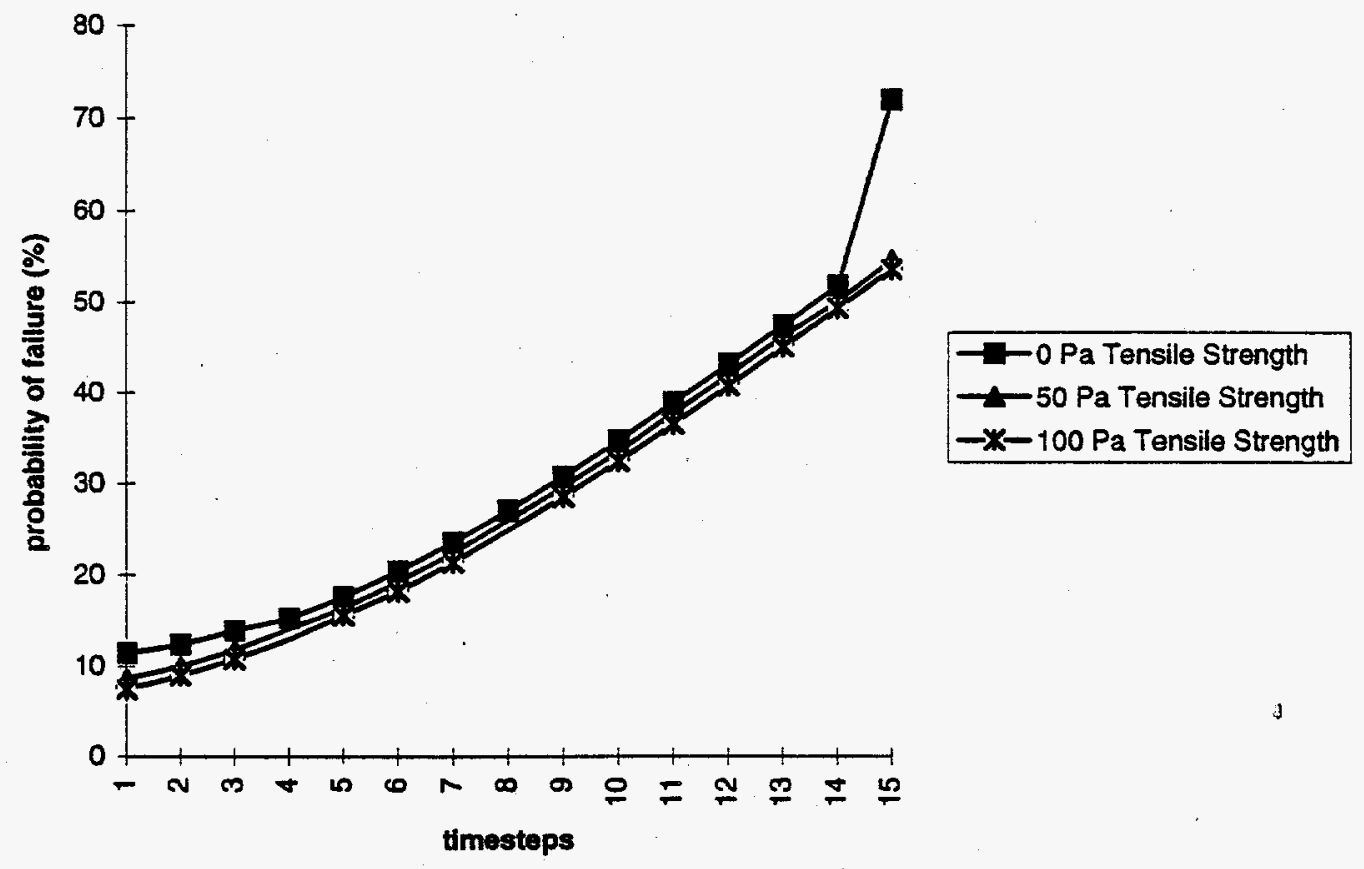

Figure 4.1. Probability of Failure Versus Time Step

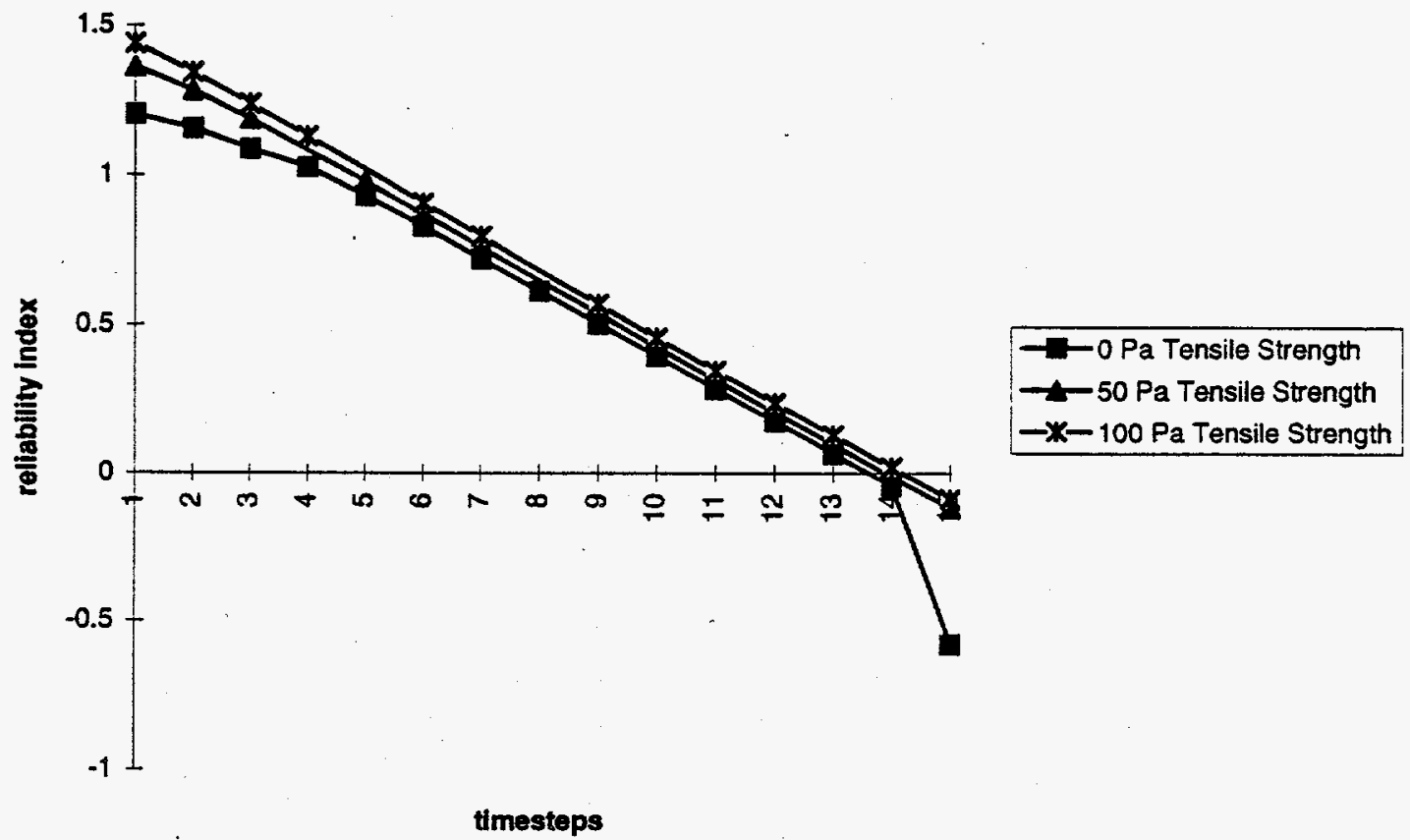

Figure 4.2. Reliability Index Versus Time Step 

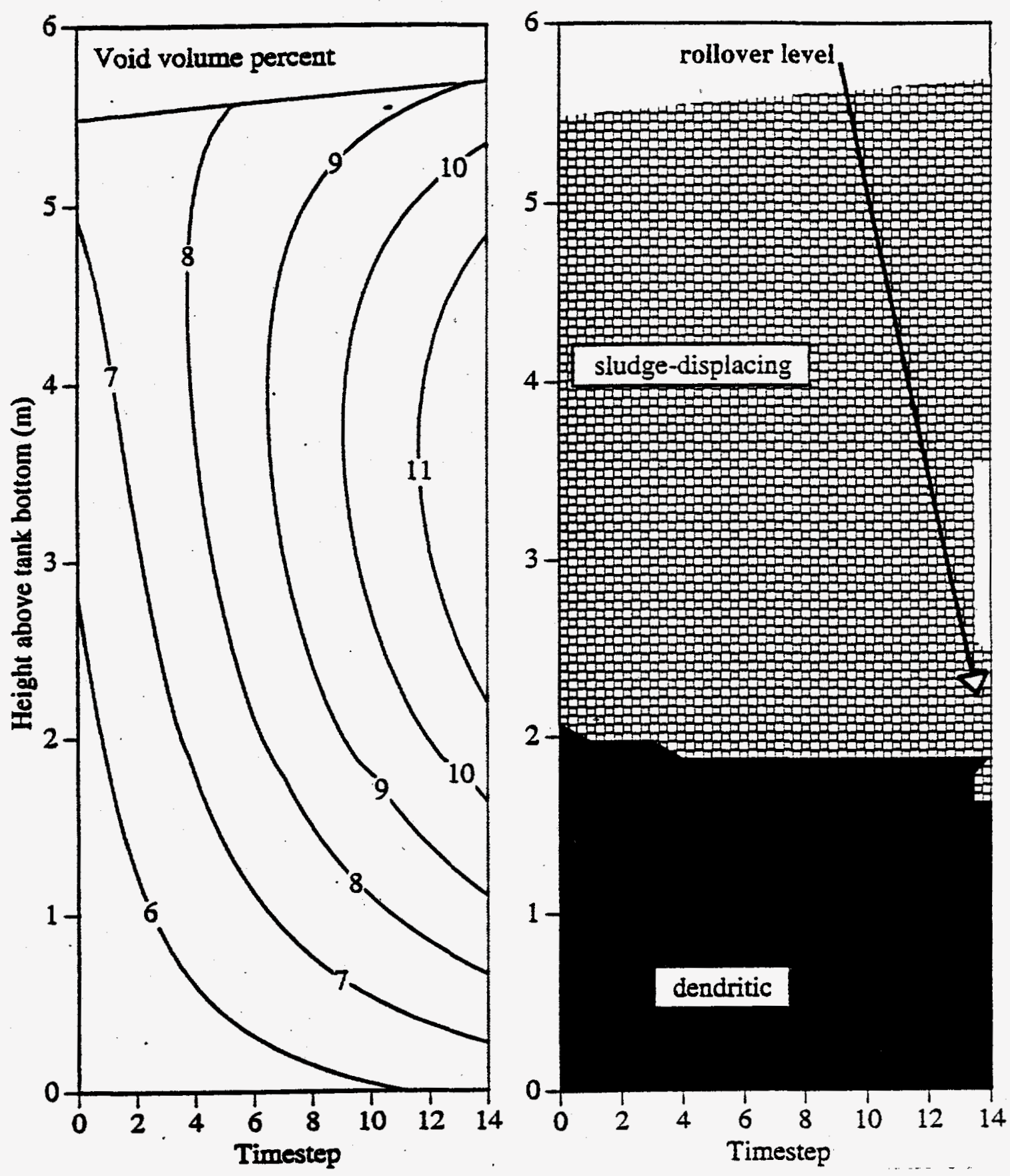

Figure 4.3. Void Percentage and Bubble Shapes for First Rollover Cycle, Tank 241-SY-101 


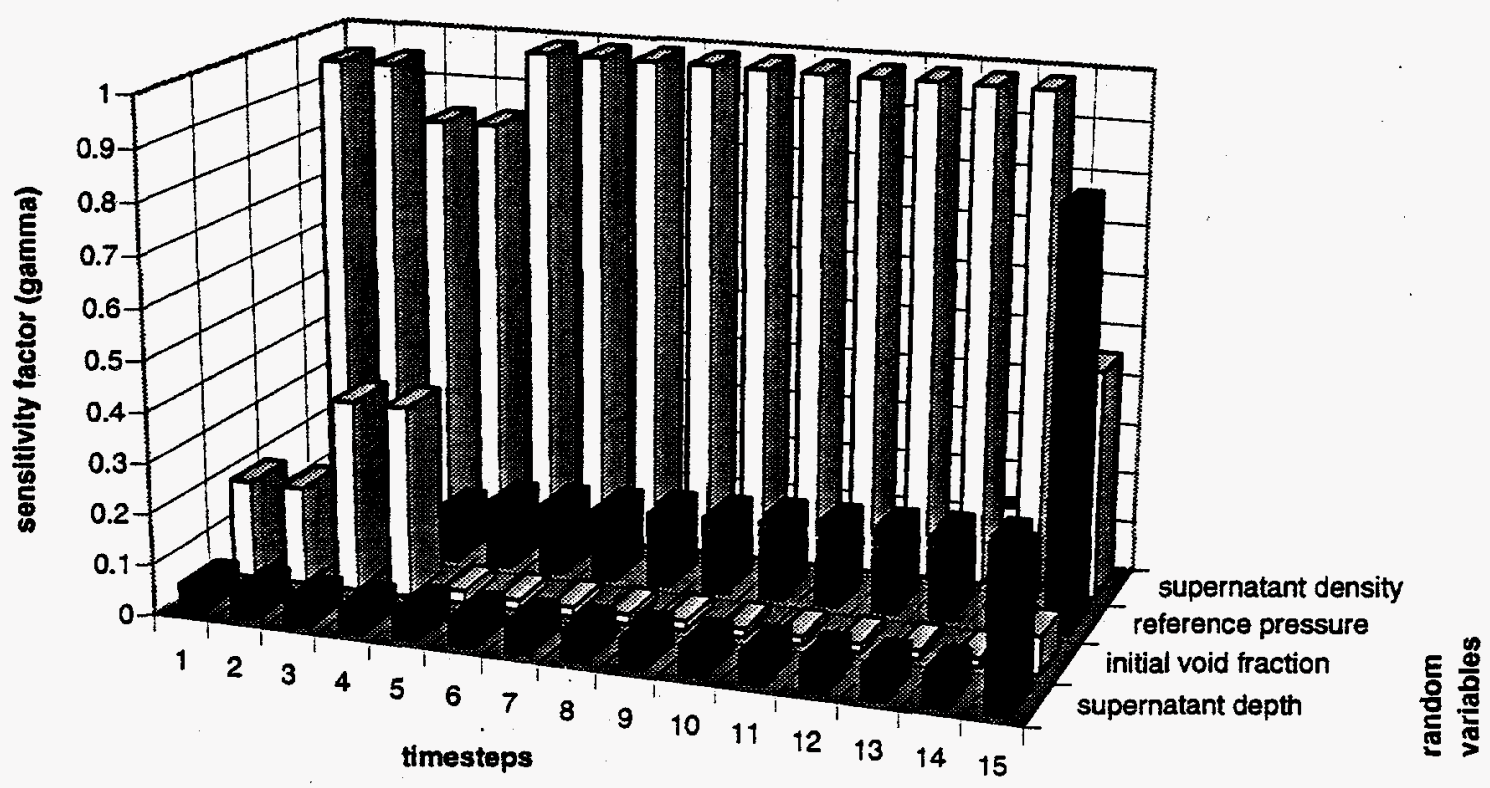

Figure 4.4. Sensitivity of Reliability Index to Random Variables for Sludge with No Tensile Strength

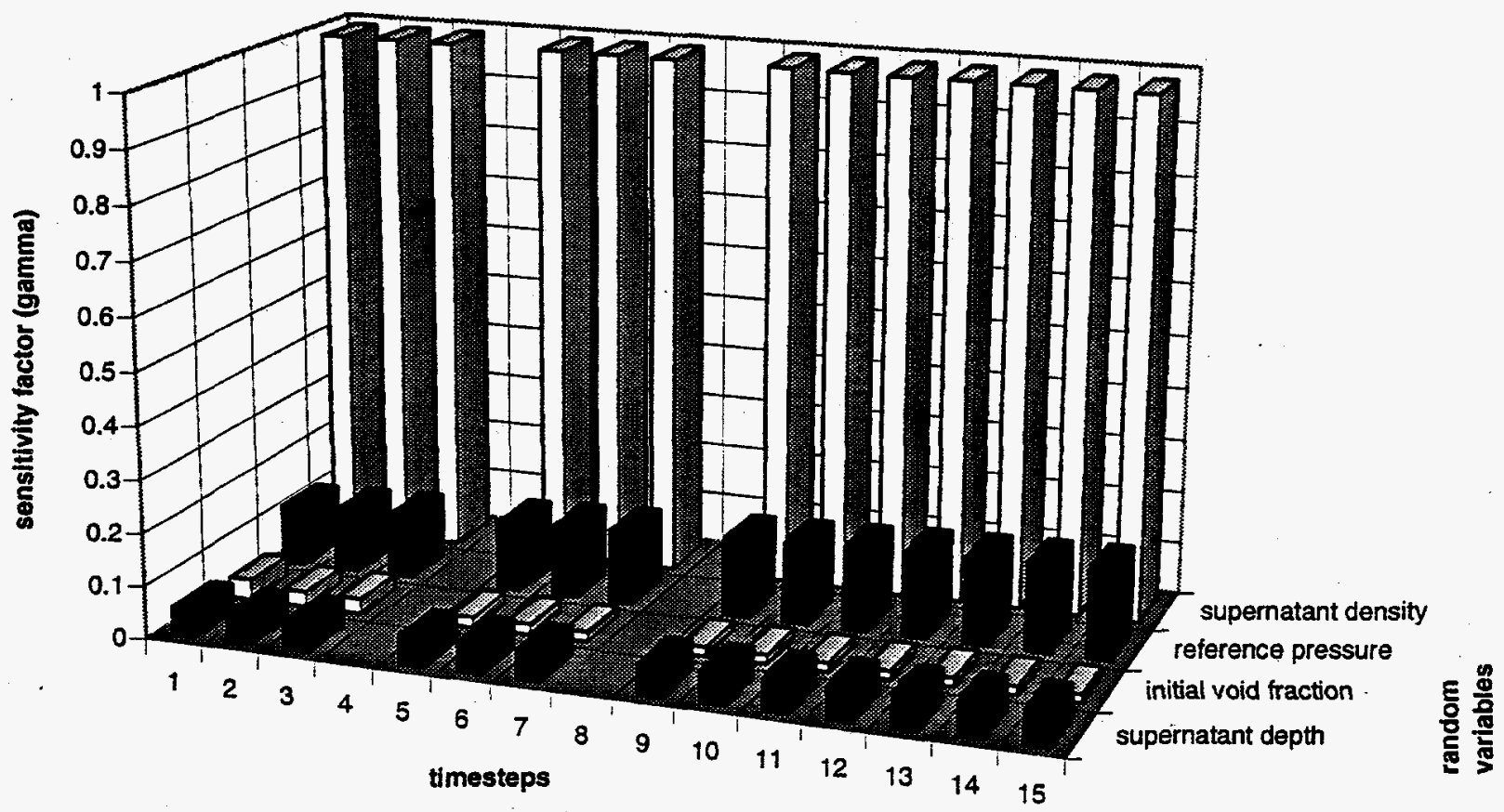

Figure 4.5. Sensitivity of Reliability Index to Random Variables for Sludge with Tensile Strength of $50 \mathrm{~Pa}$ 


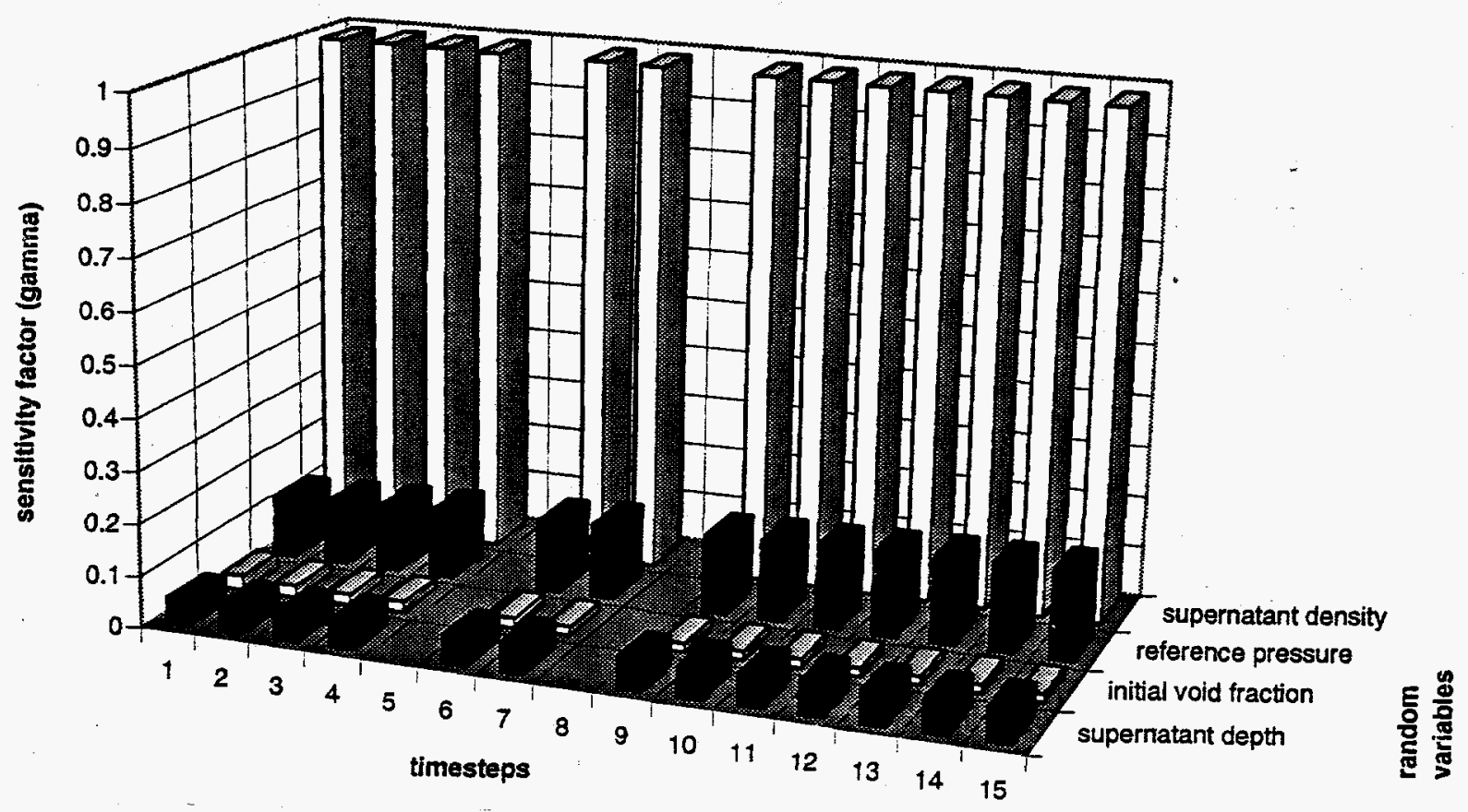

Figure 4.6. Sensitivity of Reliability Index to Random Variables for Sludge with Tensile Strength of $100 \mathrm{~Pa}$

For all steps when the sludge has strength and also for steps 4 through 14 for the nostrength case, the probability of a rollover is slightly sensitive to the pressure in the tank. This is caused by the dependence of the void fraction on both the rate of gas generation and the pressure. The solution was shown to be relatively insensitive to the supernatant depth and the initial void fraction at the top of the sludge.

The distribution of vertical stress, $\sigma_{z}$, through the depth of sludge at the third column of finite elements for the failure state at time steps 3,10 , and 15 is shown in Figures 4.7 through 4.9. In all cases, the target vertical tensile stress was met at failure, indicating that the design point does indeed reside on the limit-state surface. Again, there is an obvious difference in the mechanism of failure for steps 1 through 4 compared to that for steps 5 through 15. For the early steps, the upper portion of the sludge is either buoyant (when the sludge has strength) or about to become so (for no sludge strength) at the limit state. For failure at the later steps, the buoyant region is trapped below the sludge surface, leading to rollover. 


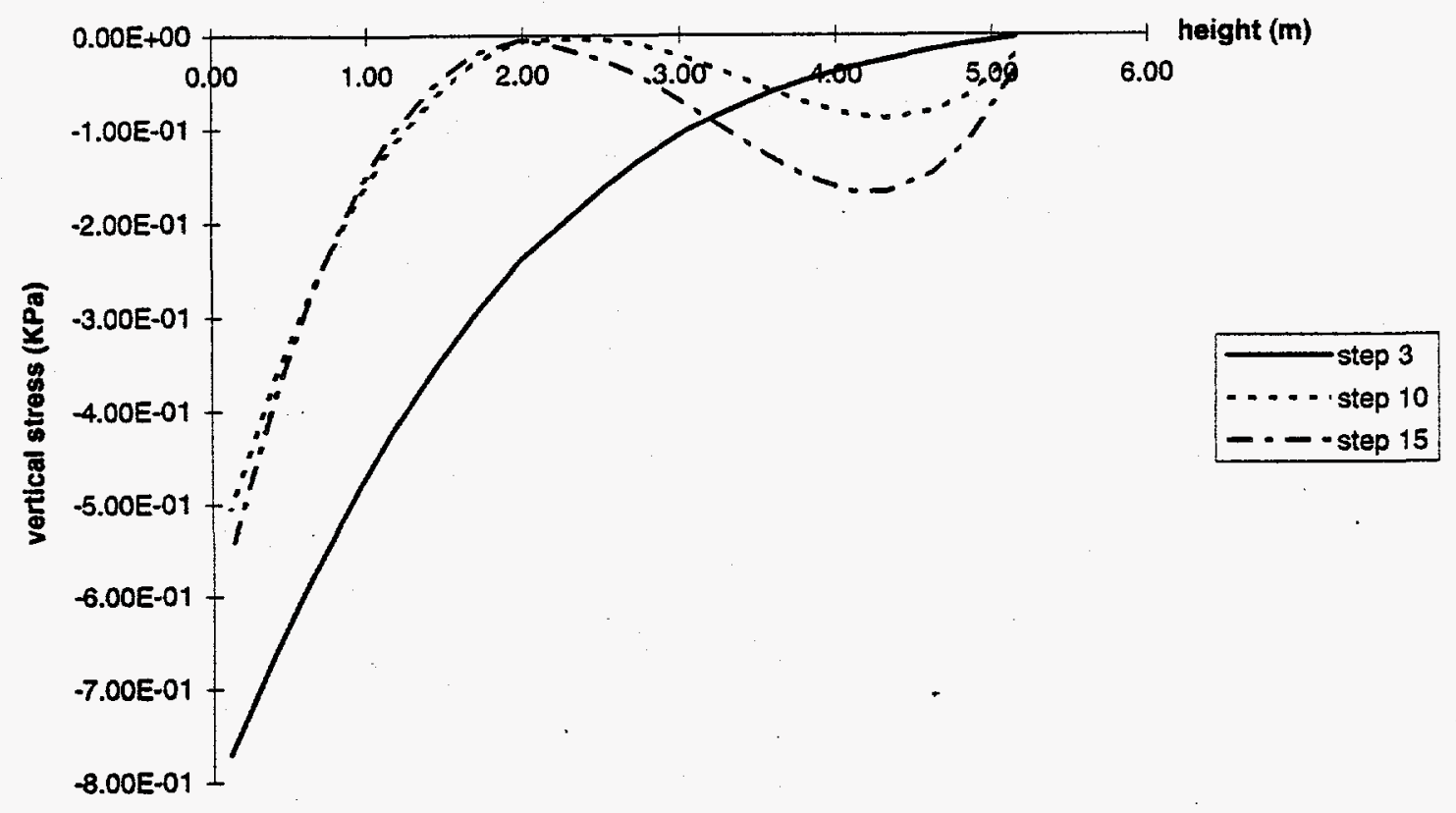

Figure 4.7. Vertical Stress Versus Height for Sludge with No Strength

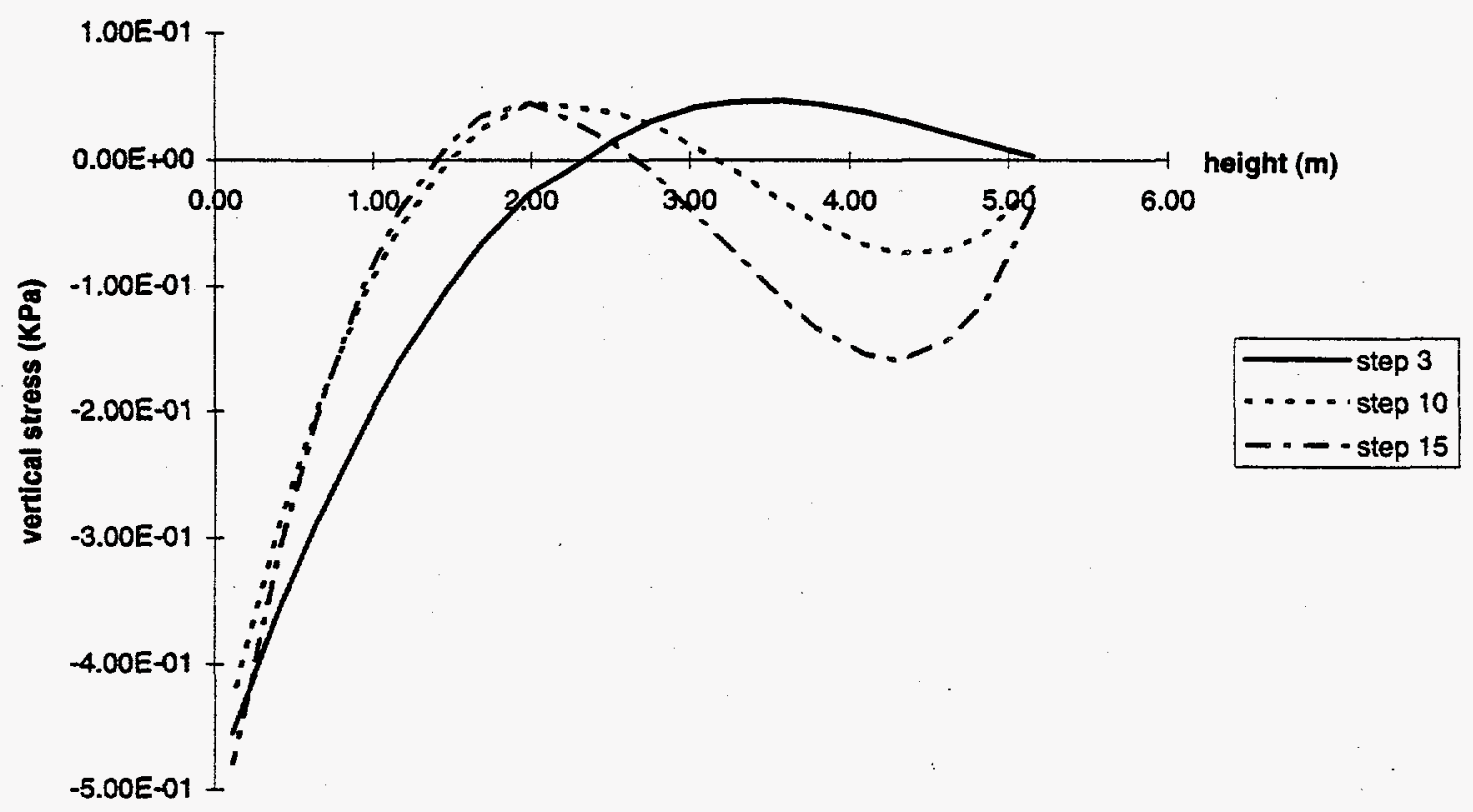

Figure 4.8. Vertical Stress Versus Height for Sludge with Tensile Strength of $50 \mathrm{~Pa}$ 


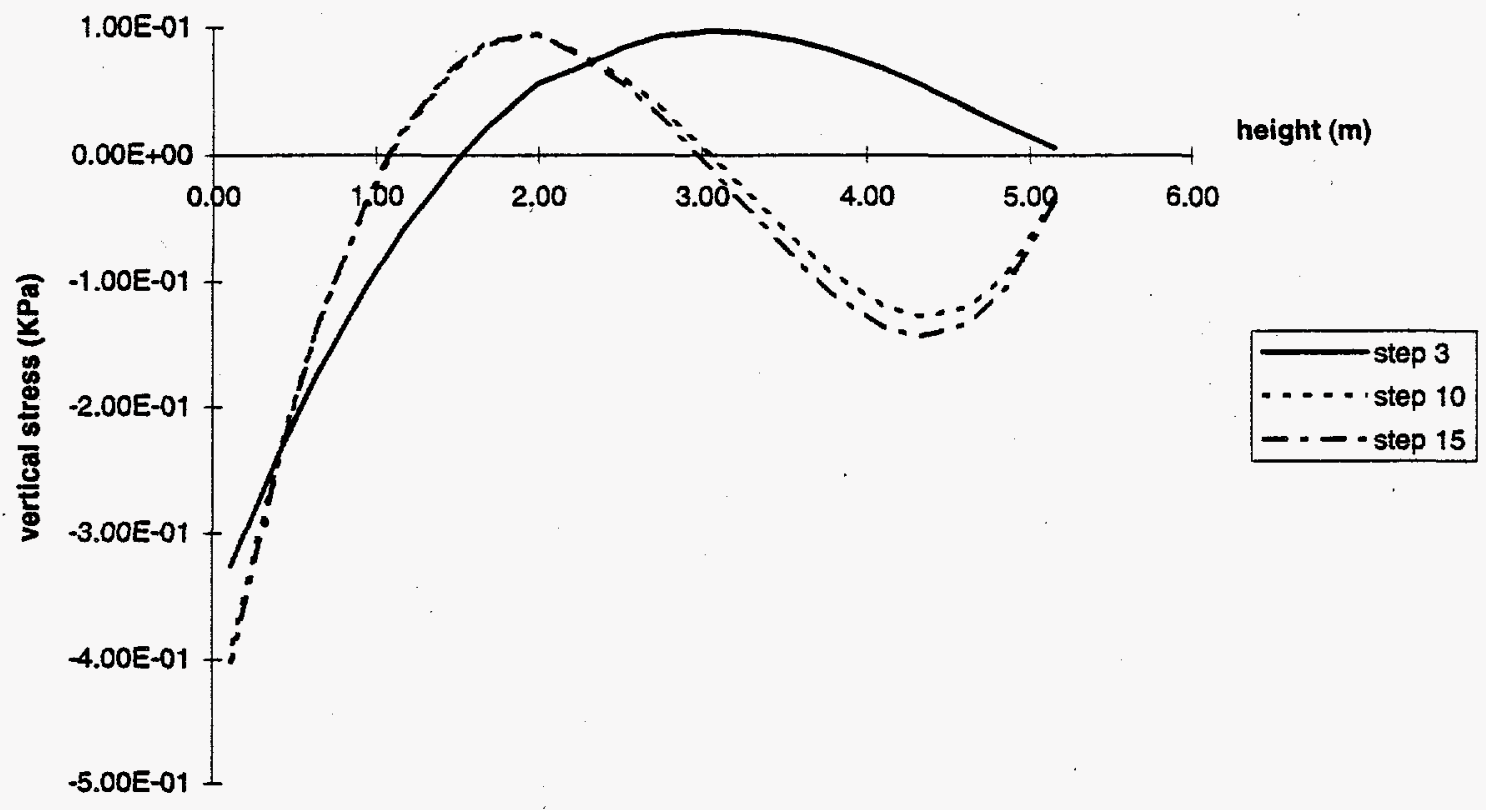

Figure 4.9. Vertical Stress Versus Height for Sludge with Tensile Strength of $100 \mathrm{~Pa}$ 


\subsection{Conclusions}

On the basis of the reliability analyses that have been completed, several conclusions can be drawn.

1. For applications in which much uncertainty exists regarding material properties, loads, and other aspects of a structure, probabilistic finite element analysis is a useful tool for quantifying the uncertainty of the behavior and for identifying parameters to which the system is sensitive. For the application described in this report, a relationship between the probability of a sludge rollover event (or, conversely, the reliability of the system) and increments of gas generation has been presented. This relationship could be used to assist in the scheduling of mitigation efforts, which could be based on target values of reliability.

2. The probability of the occurrence of a rollover event was shown to be directly affected by the density of the supernatant. However, the properties of the liquid are very difficult to control by those managing the tanks. On the other hand, of the other random variables, the solution was also shown to be relatively sensitive to the pressure in the tank. Therefore, it has been shown that the reliability of the system with respect to rollover will increase if the mean value of pressure is decreased at early stages of bubble growth and increased at later stages. This is indicated by the fact that the pressure for the design point (i.e., the point of lowest reliability) is higher than the mean value for the first time steps, decreasing steadily with time to a value below the mean at the last steps.

3. Two distinct mechanisms of failure are evident as a result of the analyses. For the early steps, the upper portion of the sludge is either buoyant (when the sludge has strength) or about to become so (for no sludge strength) at the limit state. For failure at the later steps, the buoyant region is trapped below the sludge surface, leading to rollover.

4. The strength of the sludge and its failure mechanism are still quite uncertain. Because of these uncertainties, the limit-state functions were deliberately kept simple. As more refined models are proposed, they can be implemented into the user-defined program segment and reliability analyses can be performed. For example, the enhancement of the finite element portion to include elastoplastic material behavior is recommended.

5. The reliability solution and the sensitivity information are totally dependent upon the choice of random variables and their statistical data. If accurate quantitative output is desired, the supporting input information must be 
gathered. The results of this report should be useful in defining the direction of future efforts. 


\subsection{References}

Allemann, R. T., Z. I. Antoniak, J. R. Friley, C. E. Haines, L. M. Liljegren, and S. Somasundaram. 1990a. 'Mechanistic Analysis of Double-Shell Tank Gas Release - Progress Report, November. PNL-7657, Pacific Northwest Laboratory, Richland, Washington.

Allemann, R. T., Z. I. Antoniak, J. R. Friley, C. E. Haines, L. M. Liljegren, and S. Somasundaram. 1990b. Collection and Analysis of Existing Data for Waste Tank Mechanistic Analysis - Progress Report, December. PNL-7658, Pacific Northwest Laboratory, Richland, Washington.

Allemann, R. T., Z. I. Antoniak, L. L. Eyler, and L. M. Liljegren. 1991. Conceptual Models for Waste Tank Mechanistic Analysis - Status Report, January. PNL-8011, Pacific Northwest Laboratory, Richland, Washington.

Allemann, R. T., T. M. Burke, D. A. Reynolds, and D. E. Simpson. 1993. Assessment of Gas Accumulation and Retention - Tank 241-SY-101. WHC-EP-0576, Westinghouse Hanford Company, Richland, Washington.

Cook, R. D., D. S. Malkus, and M. E. Plesha. 1989. Concepts and Applications of Finite Element Analysis. 3rd ed. John Wiley and Sons, New York.

Gauglitz, P. A., L. A. Mahoney, D. P. Mendoza, and M. C. Miller. 1994. Mechanisms of Gas Bubble Retention. PNL-10120, Pacific Northwest Laboratory, Richland, Washington.

Gauglitz, P. A., S. D. Rassat, M. R. Powell, R. R. Shah, and L. A. Mahoney. 1995. Gas Bubble Retention and Its Effects on Waste Properties: Retention Mechanisms, Viscosity, and Tensile and Shear Strengths. PNL-10740, Pacific Northwest Laboratory, Richland, Washington.

Liu, P. L., and A. Der Kiureghian. 1989. Finite-Element Reliability Methods for Geometrically Nonlinear Stochastic Structures. UCB/SEMM-89/05, Department of Civil Engineering, University of California at Berkeley, Berkeley, California.

Liu, P. L., H. Z. Lin, and A. Der Kiureghian. 1989. CALREL User Manual. UCB/SEMM-89/18, Department of Civil Engineering, University of California at Berkeley, Berkeley, California.

Tingey, J. M., P. R. Bredt, and E. H. Shade. 1994. The Effects of Heating and Dilution on the Rheological and Physical Properties of Tank 241-SY-101. PNL-10198, Pacific Northwest Laboratory, Richland, Washington. 
Trent, D. S., and T. E. Michener. 1993. Numerical Simulation of Jet Mixing Concepts in Tank 241-SY-101. PNL-8559, Pacific Northwest Laboratory, Richland, Washington. 


\section{Distribution}

Offsite

No. of

Copies

2 DOE/Office of Scientific and Technical Information

W. F. Cofer

Department of Civil and Environmental Engineering

Washington State University

Pullman, WA 99164-2910

B. S. Hudson

P. O. Box 271

Lindsborg, KA 67456

Onsite

4 DOE Richland Operations Office

J. M. Gray

S7-54

M. F. Jarvis

S7-54

G. W. Rosenwald

S7-54

Reading Room

A1-65

13 Westinghouse Hanford Company

H. Babad

S7-30

W. B. Barton

J. D. Hopkins

G. D. Johnson (5)

J. W. Lentsch

E. J. Lipke

N. G. McDuffie

D. A. Reynolds

R. J. Van Vleet
R2-11

R2-11

S7-15

S7-15

S7-14

S7-15

R2-11

H4-63
No. of

Copies

Onsite

22 Pacific Northwest Laboratory

M. E. Brewster K7-15

J. W. Brothers (5) K5-22

P. A. Gauglitz P7-41

R. T. Hallen $\quad$ P8-38

K. I. Johnson K5-26

M. A. Khaleel (5) K5-26

H. C. Reid K7-15

F. A. Simonen K5-26

C. W. Stewart K7-15

L. R. Pedersen K2-44

Technical Report Files (5)

Distr.1 